\title{
Wavelet-Based Genomic Signal Processing for Centromere Identification and Hypothesis Generation
}

\author{
Deborah Weighill 1,2, David Macaya-Sanz ${ }^{3}$, Stephen Paul DiFazio ${ }^{3}$, Wayne Joubert ${ }^{4}$, \\ Manesh Shah ${ }^{2}$, Jeremy Schmutz ${ }^{5,6}$, Avinash Sreedasyam ${ }^{6}$, Gerald Tuskan ${ }^{2}$ and \\ Daniel Jacobson ${ }^{1,2 *}$ \\ ${ }^{1}$ The Bredesen Center for Interdisciplinary Research and Graduate Education, University of Tennessee, Knoxville, TN, United \\ States, ${ }^{2}$ Biosciences Division, Oak Ridge National Laboratory, Oak Ridge, TN, United States, ${ }^{3}$ Department of Biology, West \\ Virginia University, Morgantown, WV, United States, ${ }^{4}$ Oak Ridge Leadership Computing Facility, Oak Ridge National \\ Laboratory, Oak Ridge, TN, United States, ${ }^{5}$ Department of Energy Joint Genome Institute, Walnut Creek, CA, United States, \\ ${ }^{6}$ HudsonAlpha Institute for Biotechnology, Huntsville, AL, United States
}

\section{OPEN ACCESS}

Edited by:

Ramana V. Davuluri,

Northwestern University, United States

Reviewed by:

Hao Sun

The Chinese University of Hong Kong,

China

Manoj Kandpal,

Northwestern University, United States

*Correspondence:

Daniel Jacobson

jacobsonda@ornl.gov

Specialty section:

This article was submitted to

Bioinformatics and Computational

Biology,

a section of the journal

Frontiers in Genetics

Received: 02 November 2018

Accepted: 06 May 2019

Published: 31 May 2019

Citation:

Weighill D, Macaya-Sanz D,

DiFazio SP, Joubert W, Shah M,

Schmutz J, Sreedasyam A, Tuskan G and Jacobson D (2019)

Wavelet-Based Genomic Signal

Processing for Centromere

Identification and Hypothesis

Generation. Front. Genet. 10:487.

doi: 10.3389/fgene.2019.00487
Various 'omics data types have been generated for Populus trichocarpa, each providing a layer of information which can be represented as a density signal across a chromosome. We make use of genome sequence data, variants data across a population as well as methylation data across 10 different tissues, combined with wavelet-based signal processing to perform a comprehensive analysis of the signature of the centromere in these different data signals, and successfully identify putative centromeric regions in $P$. trichocarpa from these signals. Furthermore, using SNP (single nucleotide polymorphism) correlations across a natural population of $P$. trichocarpa, we find evidence for the co-evolution of the centromeric histone $\mathrm{CENH} 3$ with the sequence of the newly identified centromeric regions, and identify a new $\mathrm{CENH} 3$ candidate in $P$. trichocarpa.

Keywords: Populus trichocarpa centromeres, wavelet transform, DNA methylation, SNP density, CENH3, coevolution, data integration

\section{INTRODUCTION}

Integrating data from multiple different sources is a task which is becoming more prevalent with the increased availability of systems biology data from high-throughput 'omics technologies and phenotyping strategies (Gomez-Cabrero et al., 2014). Developing statistical and mathematical approaches to integrate this data in order to provide an increased understanding of the biological system is thus an important endeavor. For the bioenergy feedstock crop Populus trichocarpa, several heterogenous datasets have been generated. The full genome sequence is available and is currently in its third version (Tuskan et al., 2006). A large collection of $\sim 28,000,000$ Single Nucleotide Polymorphisms (SNPs) called across 882 genotypes are publicly available (https://doi.ccs.ornl. 434gov/ui/doi/55), which were derived from the resequenced genomes of $\sim 1,000 P$. trichocarpa genotypes propagated in common gardens (Tuskan et al., 2011; Slavov et al., 2012; Evans et al., 2014). Methyl-DNA immunoprecipitation (MEDIP)-seq DNA methylation data is also available for 10 different $P$. trichocarpa tissues (Vining et al., 2012). A gene expression atlas for $P$. trichocarpa is also available on Phytozome (Goodstein et al., 2012). 
Integration of multiple heterogeneous data types requires coercing them into mathematical structures that allow them to be compared/merged/layered. For example, each of the data types mentioned above provides feature(s) which can be represented as vectors of numbers, with each vector representing a signal which varies across a chromosome, for example, the gene density across a chromosome, or the methylation profile of a chromosome. Once represented as a signal, these data types are amenable to signal processing techniques. This study aims to make use of signal processing techniques of these multiple data types in order to attempt to identify chromosome structural features in $P$. trichocarpa.

The centromere is an important chromosomal structure which controls the segregation of chromosomes during cell division, and is the location for the assembly for the kinetochore protein complex (O'Connor, 2008; Feng et al., 2015). Centromeric chromatin contains a histone $\mathrm{H} 3$ variant specific to the centromere (CENH3), which has been found in many organisms, including plants (Talbert et al., 2002). Studies by Henikoff et al. (2001) and Cooper and Henikoff (2004) have suggested that CENH3 is co-evolving with the sequence of the centromere.

Centromeric regions can vary in size, and can be small regions consisting of only one nucleosome, such as in Saccharomyces cerevisiae (Furuyama and Biggins, 2007; Feng et al., 2015), while plant centromeric regions are large ( $\mathrm{Mb}$ scale), and consist of repetitive sequences (Mehrotra and Goyal, 2014; Feng et al., 2015). Centromeres also have epigenetic characteristics in that plant centromeric regions have been found to be relatively highly methylated (Zhang et al., 2006; Vining et al., 2012).

Previously, putative centromere positions were identified in $P$. trichocarpa as chromosomal regions of low gene density and high methylation, presented visually, but coordinates were not reported (Vining et al., 2012). Putative centromere positions have also been identified based on recombination rates along chromosomes through visual inspection of profiles of $4 \mathrm{~N}_{e} \mathrm{c}$ (Slavov et al., 2012). Cossu et al. (2012) identified putative centromeric repeats of $P$. trichocarpa which identified putative centromere positions on some of the $P$. trichocarpa chromosomes in a previous assembly of the genome. In Pinosio et al. (2016), putative centromeres were identified as regions as the $250 \mathrm{~kb}$ window on each chromosome with the lowest gene density, and reported enrichment of insertions and repetitive elements in the centromeric regions. However, to our knowledge, there has not been a comprehensive study of $P$. trichocarpa centromeres integrating various available data types and multiple lines of evidence.

The large collection of data available for $P$. trichocarpa provides a source of multiple features which can be represented as density signals across each chromosome. Certain features, such as gene density and SNP density, can be readily constructed from the data available. Other lines of evidence, such as SNP correlation/co-segregation need to be calculated from the data before the chromosome signals can be constructed.

Such chromosome signals contain variation on multiple scales, including high frequency (narrow) peaks and lowfrequency (broad) peaks. These different scales of peaks contain different information. Thus, techniques to analyse these signals at different scales are valuable (see Spencer et al., 2006; McCormick et al., 2017). The Wavelet Transform, a signal processing technique, can be used to unpack the information in different scales of a signal, such as a density profile across a chromosome (Spencer et al., 2006). In general, the wavelet transform involves expressing a function (signal) as a linear combination of functions called wavelets. These functions are scaled translations of a mother wavelet, such as the Ricker Wavelet (Figure 1). What results from a wavelet transform is a wavelet coefficient $W(s, \tau)$ (Equation 1), for every scale $s$ and translation (shift along the $\mathrm{x}$-axis) $\tau$ (Leavey et al., 2003).

$$
W(s, \tau)=\frac{1}{\sqrt{s}} \int f(t) \psi^{*}\left(\frac{t-\tau}{s}\right) d t
$$

Given the peak-like shape of the wavelet, a wavelet coefficient will indicate "how much of a peak" is present at a particular scale and at a particular position of the signal. Thus, the wavelet transform allows us to investigate the peaks of a signal at different scales and locations.

This study makes use of the Continuous Wavelet Transform (CWT) in characterizing chromosomal gene density, SNP density and methylation density signals in $P$. trichocarpa. We use the resulting CWT coefficient landscapes to identify the putative centromere locations and illustrate the wavelet signature of a centromere. We also investigate potential co-evolution signatures between the centromeric histone $\mathrm{CENH} 3$ and the newly identified centromeric regions through the calculation of SNP correlations across the population, and find evidence supporting the hypothesis of the co-evolution of putative $P$. trichocarpa CENH3 genes with the centromere sequences in $P$. trichocarpa. While wavelets have previously been used in chromosome classification (Wu and Castleman, 2000), and the discrete wavelet transform has been used in the analysis of feature profiles across a chromosome in human (Spencer et al., 2006), to our knowledge this work presents the first use of the continuous wavelet transform in the identification of centromere positions from SNP and methylation density profiles. This study provides an example of how signal processing of multiple data types can be used to generate hypotheses surrounding the structure of chromosomes.

\section{METHODS AND MATERIALS}

\subsection{Variant Data and SNP Correlations}

Populus trichocarpa (Tuskan et al., 2006) variant data (doi: 10. 13139/OLCF/1411410) was obtained from https://doi.ccs.ornl. 434gov/ui/doi/55. This dataset consists of SNP 28,342,758 SNPs called across $882 P$. trichocarpa genotypes and is derived the whole genome resequencing of a Genome Wide Association Study (GWAS) population clonally replicated in common gardens (Tuskan et al., 2011).

The most reliable SNPs within the dataset were selected, consisting of the $90 \%$ tranche (the tranche recovering $90 \%$ of the "true" SNPs). VCFtools (Danecek et al., 2011) was used to extract the desired Tranche of SNPs from the VCF file and reformat 

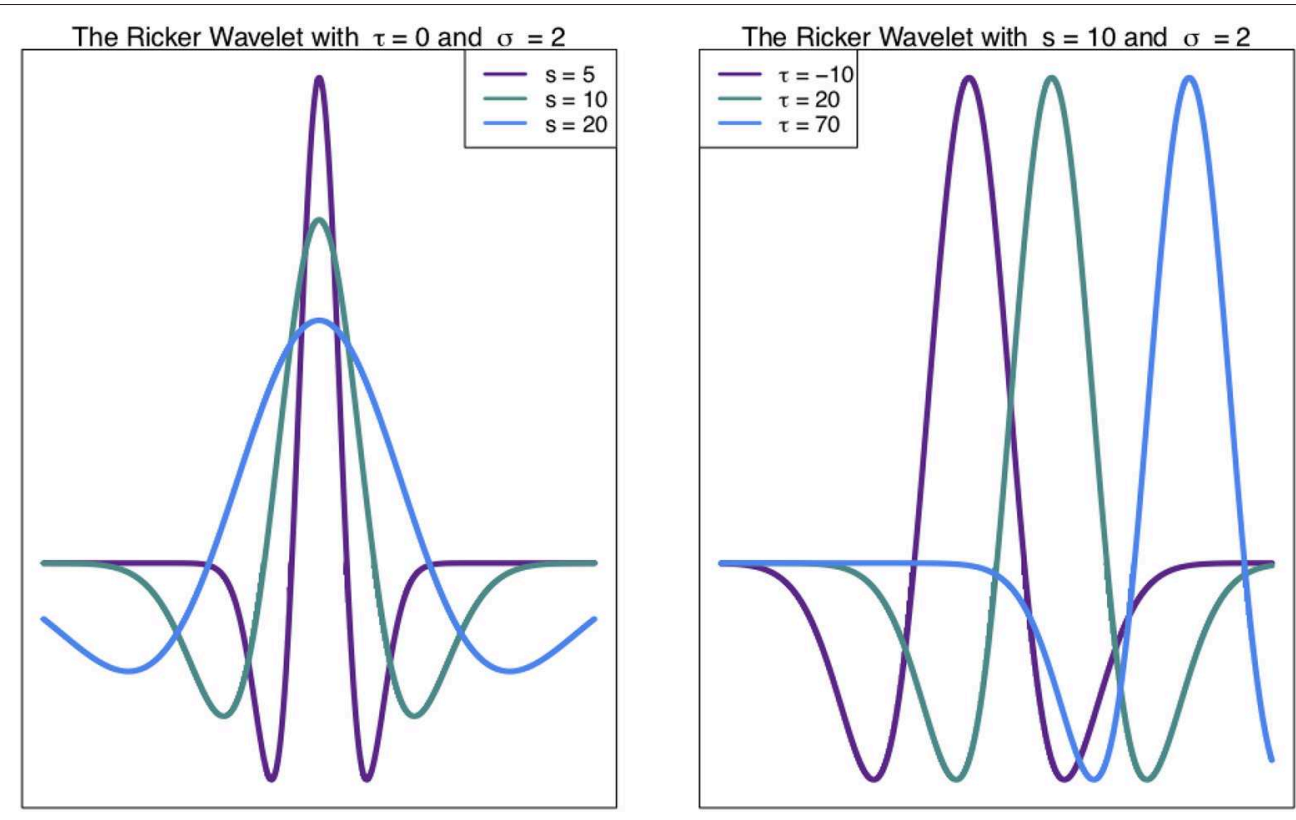

FIGURE 1 | Ricker Wavelet. The Ricker wavelet shown for different values of scale $s$ and translation $\tau$ in Equation (1) (Leavey et al., 2003; Machado et al., 2011).

it into .tfam and .tped files. Plink (Purcell et al., 2007, http:// pngu.mgh.harvard.edu/purcell/plink/) was used to determine the minor allele frequency (MAF) and the call rate (fraction alleles observed) for each SNP, and removed all SNPs with MAF $\leq 0.01$ and call rate $\leq 0.5$.

Correlations between all pairs of SNPs were calculated using the Custom Correlation Coefficient (CCC) (Climer et al., 2014a,b). This was performed on both the filtered set of SNPs as well as the entire $90 \%$ tranche, using a new, GPU implementation of the CCC metric for the calculation of SNP correlations (Joubert et al., 2017) as well as the original software (Climer et al., 2014a,b), respectively. Calculation of the CCC between all pairs of SNPs using the original software was performed in parallel, as described in Weighill et al. (2018). Briefly, the CCC between allele $x$ at location $i$ and allele $y$ and location $j$ is defined as:

$$
\operatorname{CCC}_{i_{x} j_{y}}=\frac{9}{2} R_{i_{x} j_{y}}\left(1-\frac{1}{f_{i_{x}}}\right)\left(1-\frac{1}{f_{j_{y}}}\right)
$$

where $R_{i_{x} j_{y}}$ is the relative co-occurrence of allele $x$ at location $i$ and allele $y$ at location $j, f_{i_{x}}$ is the frequency of allele $x$ at location $i$ and $f_{j y}$ is the frequency of allele $y$ at location $j$.

This was performed in a parallel fashion by constructing a Perl wrapper around the ccc binary, making use of the Parallel::MPI::Simple Perl module, developed by Alex Gough and available on The Comprehensive Perl Archive Network (CPAN) at www.cpan.org. "The set of $\sim 10$ million SNPs was divided into 20 different blocks, and the CCC was calculated for each withinblock and cross-block comparison in separate jobs, to a total of 210 MPI jobs ... A threshold of 0.7 was then applied." (Quotation from Weighill et al., 2018).

\subsection{Chromosome Feature Profile Construction}

\subsubsection{SNP Density Profiles}

A SNP density profile was created for each chromosome using the filtered set of SNPs by counting the number of these SNPs in non-overlapping $10 \mathrm{~kb}$ windows across the chromosome.

\subsubsection{Methylation Profiles}

Methylation (MeDIP-seq) data from 10 P. trichocarpa tissues generated from the study by Vining et al. (2012) re-aligned to the version 3 assembly of $P$. trichocarpa was downloaded from Phytozome (Goodstein et al., 2012). This data consists of MeDIP-seq reads from tissues including bud, callus, female catkin, internode explant, leaf, make catkin, phloem, regenerated internode, root and xylem tissue.

Samtools (Li et al., 2009) was used to view the data and BamTools stats (Barnett et al., 2011) was used to investigate statistics of the reads in the bam files. BEDTools (Quinlan, 2014) was used to count the number of reads mapped to 10 $\mathrm{kb}$ windows across the genome. This will allow us to construct a "mapped read density" distribution for each tissue and each chromosome, showing the number of reads which mapped to different regions of the genome, and thus indicating methylation hotspots. The BEDOPs (Neph et al., 2012) software was used to convert .gtf files of the $10 \mathrm{~kb}$ windows per chromosome into .bed files. GNU-Parallel (Tange, 2011) was used to run the BEDTools jobs in parallel.

\subsubsection{Gene Density Profiles}

Gene density profiles were constructed for each chromosome. Gene density for a given window was defined as the number of nucleotide positions within that window that reside 
within genes. Gene boundaries were determined from the Ptrichocarpa_210_v3.0.gene.gff3 annotation file obtained from the $P$. trichocarpa version 3 genome annotation (Tuskan et al., 2006) available on Phytozome (Goodstein et al., 2012) through the genome portal of the Department of Energy Joint Genome Institute (Grigoriev et al., 2011; Nordberg et al., 2014).

\subsubsection{Genome Gap Density Profiles}

Genome gap density profiles were constructed for each chromosome, similar to the approach for constructing SNP density profiles. For each non-overlapping $10 \mathrm{~kb}$ window on a chromosome, the number of " $\mathrm{N}$ " positions were counted in the genome assembly file ptrichocarpa_210_v3.0.fa obtained from the version 3 genome assembly (Tuskan et al., 2006) available on Phytozome (Goodstein et al., 2012).

\subsection{Continuous Wavelet Transform of Chromosome Feature Profiles}

The CWT was performed on chromosome feature density profiles using the wmtsa $\mathrm{R}$ wavelet package (Percival and Walden, 2006; Constantine and Percival, 2016), the R programming language (R Core Team, 2015), RStudio (RStudio Team, 2016), and various $R$ packages and resources (Constantine et al., 2016). The CWT results in sets of wavelet coefficients at different scales. These were plotted as a heatmap/coefficient landscape, showing the numerical values of the different wavelet coefficients across the signal, at different scales. Plots were generated using custom R scripts and R packages (Neuwirth, 2014; R Core Team, 2015; Nychka et al., 2017).

\subsection{Centromere Position Identification}

Putative centromeres were located for each chromosome by computationally identifying the "tooth-X-ray" signature in the wavelet landscapes. Let the matrix $M$ represent the methylation wavelet landscape and let $S$ represent the SNP wavelet landscape for a given chromosome. We identified the maximum wavelet coefficient in the upper third of the methylation wavelet landscape (internode explant tissue), and identified the scale $p$ (row of $M$ ) at which this maximum coefficient was found. This identified the general pericentromeric scale. The borders of the approximate pericentromeric regions $b_{1}$ and $b_{2}$ were identified as the zeroes of the methylation wavelet coefficient vector at scale $p$ (Supplementary Note S1, Figure S1). The minimum wavelet coefficient in the lower two thirds of $S$ between the borders $b_{1}$ and $b_{2}$ was then identified, and the scale $c$ (row of $S$ ) at which this minimum occurs was considered the centromeric scale. The methylation pericentromeric scale vector $M_{p}$ (row $p$ in matrix $M$ ) and the SNP centromeric scale vector $S_{c}$ (row $c$ of matrix $S$ ) were extracted, and scaled to have mean 0 and standard deviation 1 . The approximate centromere locations were then identified as the position $x$ at which the maximum

$$
\max \left(M_{p, x}^{*}-S_{c, x}^{*}\right)
$$

is obtained, where $M_{p, x}^{*}$ and $S_{c, x}^{*}$ represent the $x$ th entry in the scaled vectors of $M_{p}$ and $S_{c}$, respectively.

See Supplementary Note S1 and Figure S1 for further details.

\subsection{Centromere Repeat Sequence Profiles}

Plant centromere repeat sequences were downloaded from the PGSB Repeat Database (Nussbaumer et al., 2012) at http:// pgsb.helmholtz-muenchen.de/plant/recat/index.jsp. The repeat sequences were then BLASTed (Altschul et al., 1990) against the P. trichocarpa version 3 genome on Phytozome (Goodstein et al., 2012), using an E-value threshold of $10^{-5}$ and other default parameters. A density profile of BLAST hits was then constructed for each chromosome. The BLAST hit density for a given $10 \mathrm{~kb}$ window was defined as the number of positions within the window that lay within a BLAST hit (E-value $\leq$ $10^{-5}$ ) with a plant centromeric repeat sequence. We obtained putative $P$. trichocarpa centromeric repeat sequences from Cossu et al. (2012), and constructed a BLAST hit density profile for these repeat sequences in a similar manner. These centromere repeat density profiles were visualized alongside of the predicted putative centromere positions.

\subsection{Synteny Analysis}

Syntenic blocks within the $P$. trichocarpa version 3.0 genome were constructed using CoGe SynMap (Lyons et al., 2008; Haug-Baltzell et al., 2017). Syntenic segments were computed based on gene order, within a maximum of 10 non-matching genes between matching genes, and a minimum of five aligned genes per segment, similar to the parameters used in the syntenic block analysis of the original genome (Tuskan et al., 2006). Synonymous substitution rates (Ks) were also calculated. Syntenic blocks were visualized using Circos (Krzywinski et al., 2009). For each chromosome, syntenic blocks which overlapped with putative centromere locations on a chromosome were extracted.

\subsection{Co-expression Network}

Gene co-expression relationships were queried on PhytoMine though Phytozome (Goodstein et al., 2012; Kalderimis et al., 2014). A custom co-expression network was also created as described in Weighill et al. (2018) using the P. trichocarpa (Nisqually-1) RNA-seq dataset from JGI Plant Gene Atlas project (Sreedasyam et al., unpublished). This dataset consists of samples for standard tissues (leaf, stem, root and bud tissue) and libraries generated from nitrogen source study. A list of sample descriptions was accessed from Phytozome at https://phytozome. jgi.doe.gov/phytomine/aspect.do?name=Expression. Networks were visualized in Cytoscape (Shannon et al., 2003).

\subsection{Co-evolution of Putative CENH3 Genes}

The genomic sequence of the Arabidopsis thaliana CENH3 gene (AT1G01370) was obtained from Phytozome (Goodstein et al., 2012) and BLASTed against the P. trichocarpa version 3 genome (Tuskan et al., 2006) on Phytozome using default parameters. Two BLAST hits were obtained, one gene on chromosome 14 (Potri.014G096400) and one on chromosome 2 (Potri.002G169000). While Potri.014G096400 contains functional annotations on Phytozome, including Panther PTHR11426:SF46 ("Histone H3-like centromeric protein A") and Pfam PF00125 ("Core histone H2A/H2B/H3/H4SNPs"), Potri.002G169000 contains no functional annotations, likely 
because of sequencing/assembly issues. There are various exons predicted in the gene which are not considered to be translated. However, when searching for domains in the genome sequence of Potri.002G169000 using CD-search at NCBI (Marchler-Bauer and Bryant, 2004; Marchler-Bauer et al., 2010, 2014), Pfam PF00125 ("Core histone $\mathrm{H} 2 \mathrm{~A} / \mathrm{H} 2 \mathrm{~B} / \mathrm{H} 3 / \mathrm{H} 4 \mathrm{SNPs}$ ") is identified in the sequence. Thus, we have two valid CENH3 candidates. SNPs which correlated with SNPs within these genes (CCC $\geq 0.7$ ) were extracted from the SNP correlations. Density profiles of these SNPs were then constructed for all chromosomes in non-overlapping $10 \mathrm{~kb}$ bins, similar to the profile construction described above.

\section{RESULTS AND DISCUSSION}

\subsection{Chromosome Feature Profiles and CWT Coefficient Landscapes}

Chromosomal features including SNPs, genes, genome gaps and DNA methylation plotted as density signals across a chromosome result in signals that vary along the length of the chromosome (Figure 2, Figures S2-S20). These profiles show the frequency of a particular feature in $10 \mathrm{~kb}$ bins across each chromosome. These profiles vary on different scales, in that they contain peaks and valleys of different frequencies/broadness. Each of these signals has fine variation in the form of narrow, high frequency peaks, as well as broad, low-frequency peaks, as illustrated in the feature density profiles of chromosome 2 (Figure 2). The highlighted region in Figure 2 indicates the most prominent broad-scale feature, consisting of a large-scale valley in the SNP and gene density profiles, and a large-scale peak in the methylation (MeDIP-Seq read density) profile.

These large-scale peak-valley combinations of SNP, gene and methylation density profiles are observed easily on all chromosomes (Figure 3). One can see a large-scale peak in the methylation profile coinciding with valleys in the gene density and SNP density signals on each chromosome. The locations of these large-scale peak-valley combinations seem to agree with the putative $P$. trichocarpa centromere positions proposed by Vining et al. (2012) on the basis of high methylation read coverage, high repeat-to-gene ratios and recombination valleys, and also agrees with some of the putative centromere positions identified through repeat elements (Cossu et al., 2012).

The wavelet transform was used to characterize these signals at different scales, identifying peaks of different sizes. Applying the continuous wavelet transform (CWT) to such density signals results in a coefficient landscape for each signal, represented as a heatmap (Spencer et al., 2006) (Figures 4, 5B,C). The $\mathrm{x}$ axis of a coefficient landscape represents the position along the chromosome signal and the y-axis represents the scale, with small scales (high frequency peaks) at the bottom and large scales (low frequency peaks) at the top. A wavelet coefficient is calculated for each signal position and each scale, thus resulting in a landscape. The wavelet coefficient landscapes clearly illustrate the detection of the large scale peaks (blue regions) and large scale valleys (red regions) in the upper half of the landscapes, corresponding to the visible large peaks and valleys of the signals. Plotting the wavelet coefficients at a particular scale shows the smoothed peaks and troughs of the signal at that scale (Figure 5A).

\subsection{Wavelet Coefficient Landscape Signature of the Centromere}

Identification of approximate centromere locations from gene density, SNP density and methylation wavelet landscapes requires knowledge of what patterns to look for. From the literature, we know that studies in Arabidopsis have found high methylation in the centromeric/pericentromeric regions (Zhang et al., 2006), and found centromeric regions to be gene-sparse (Copenhaver et al., 1999). Similar conditions were found in $P$.

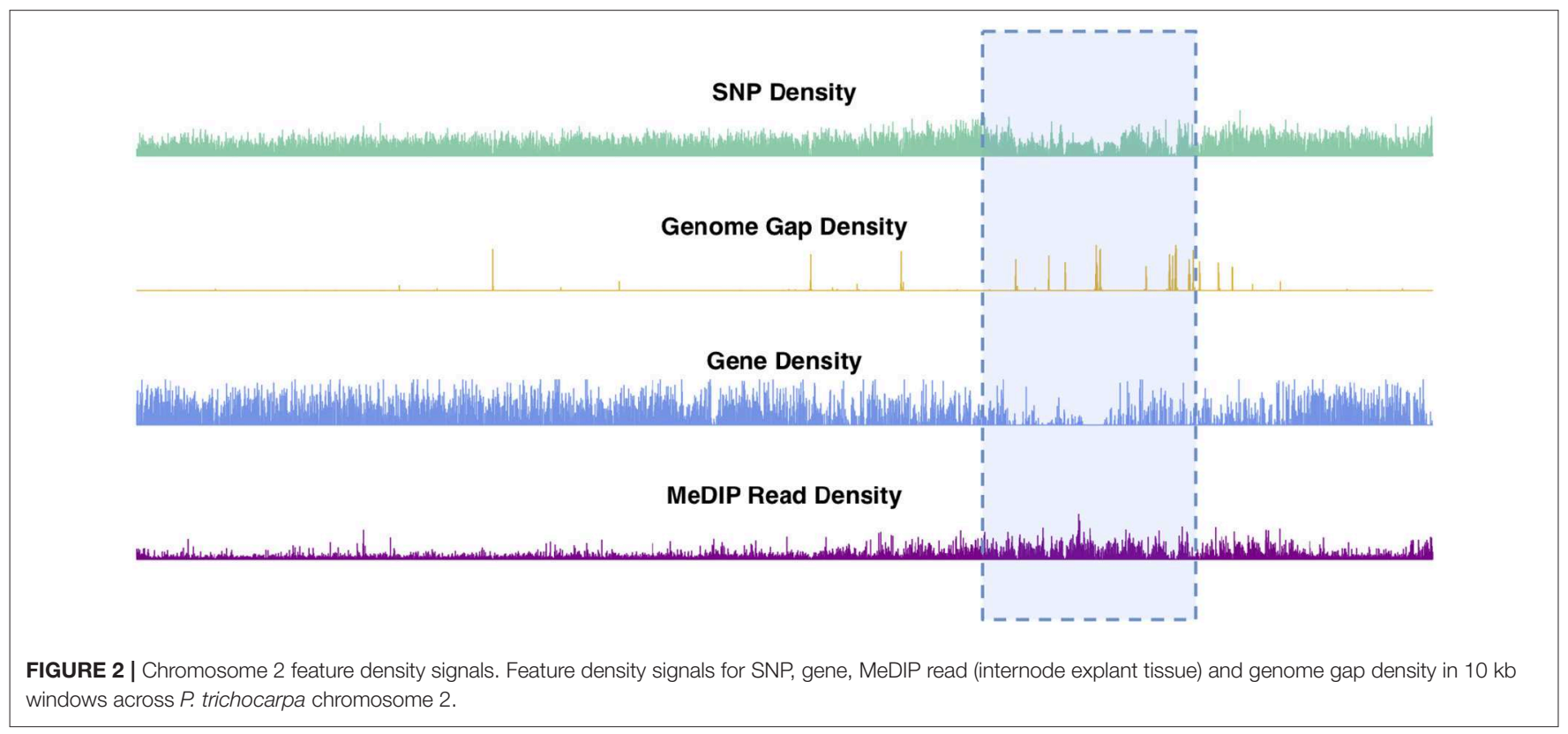




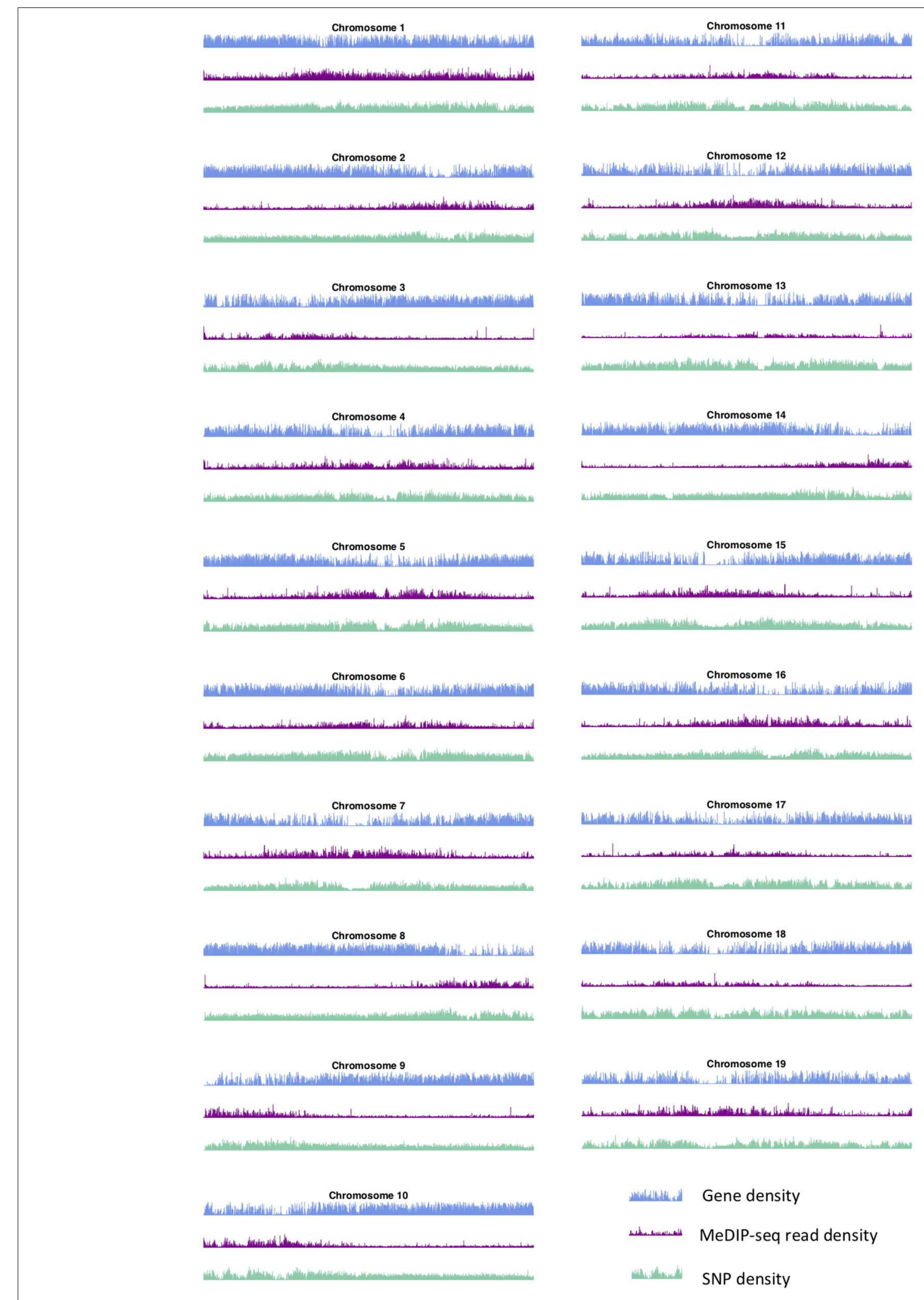

FIGURE 3 | Methylation, SNP, and gene density. SNP, gene, and methylation (internode explant tissue) density profiles for all chromosomes of $P$. trichocarpa. 


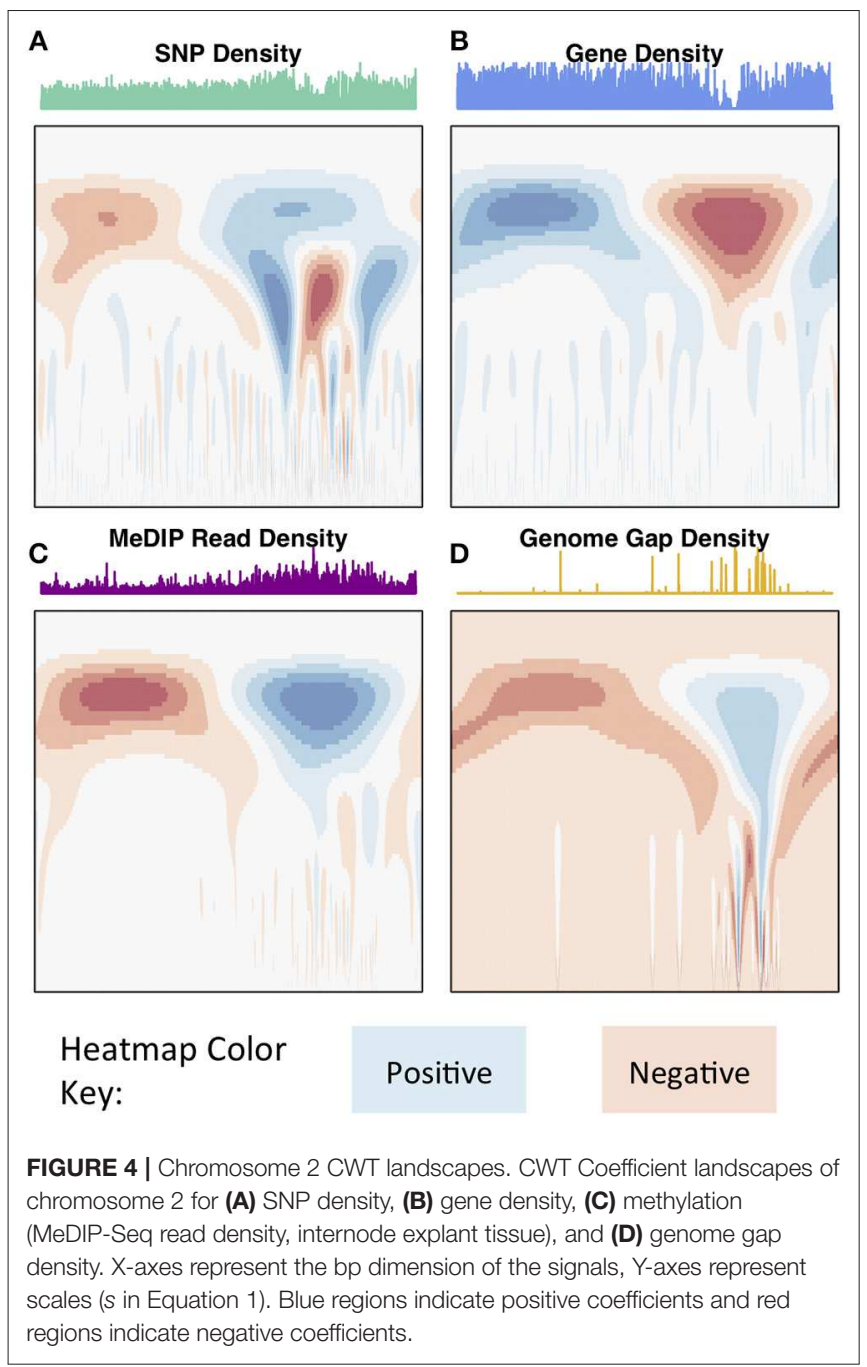

trichocarpa (Vining et al., 2012; Liang et al., 2014). Though centromeric/pericentromeric regions as a whole are highly methylated, it has been found in Maize that the active centromere consists of repeats associated with CENH3 (the modified histone found in the active centromere) and is usually less methylated when compared to the pericentromeric regions (Zhang et al., 2008). A similar pattern can be observed in Arabidopsis (Zhang et al., 2006). Figure 6 shows the methylation CWT coefficient landscapes for each chromosome in internode explant tissue. One can clearly see the large-scale peaks in each chromosome indicated by the blue regions near the top of each profile, which correspond to the broad centromeric/pericentromeric regions. In 15 of the 19 chromosomes (chromosomes 1, 2, 4, 5, 6, 7, 8, 9, 10, $13,14,16,17,18,19)$ we see evidence for the lowered methylation in the actual centromere when compared to the pericentromeric regions. In the coefficient landscapes, this is indicated by a medium-scale valley (red area) within and below the center of the large-scale peak, creating a "tooth-X-ray" like pattern (Figure 7). These centromeric wavelet coefficient signatures can also be seen in the methylation profiles of callus, female catkin, male catkin, leaf, phloem, regenerated internode, root and xylem

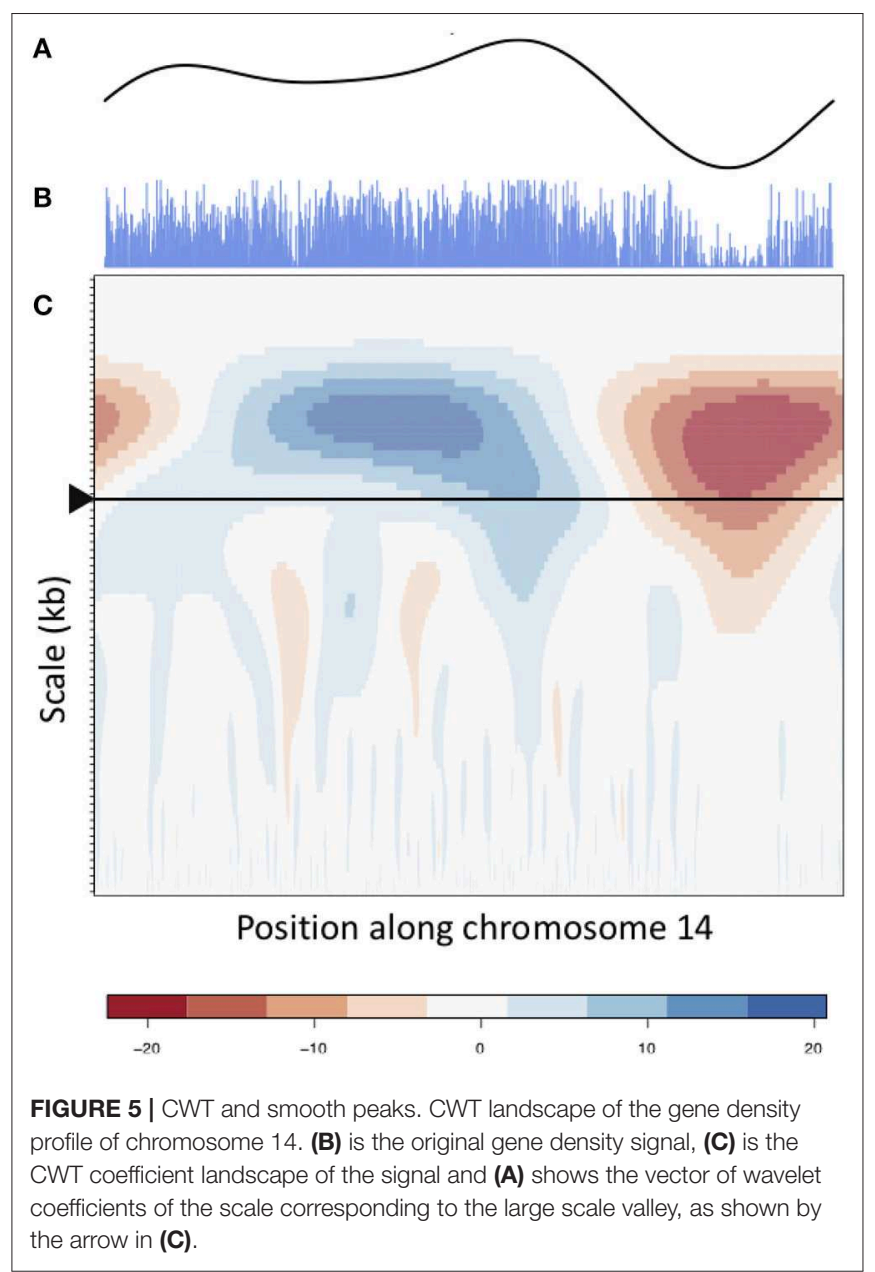

tissues (Figures S26-S34), but are mostly not visible in bud tissue (Figure S25).

SNP density has been found to be higher in the pericentromere in Arabidopsis (Ossowski et al., 2010) and lower SNP density has been found in centromere regions in sorghum (Bekele et al., 2013). The SNP wavelet landscapes for all chromosomes all contain the "tooth-X-ray" like shape, indicating a medium-scale valley in SNP density within a large-scale peak (Figure S23). The location of this signature coincides with the large-scale peak in methylation (Figures S26-S34) and valley in gene density (Figure S22), known to be characteristic of centromeric locations. As with the methylation density, this "tooth-X-ray" shape could be indicating the pericentromeric and centromeric regions of the chromosome.

It is important to consider gaps in the assembled genome (Figure S24) when interpreting chromosome density signals, because valleys in a density signal, such as SNP density, could be a meaningful biological signature (such as the centromere), or could be an artifact arising from a gap in the genome. Observing the density signals for all chromosomes (Figures S2S20) and their wavelet landscapes (Figures S22-S34) one can see that in a few chromosomes, (for example, chromosome 18) the largest genome gap co-locates with the largest valley in 

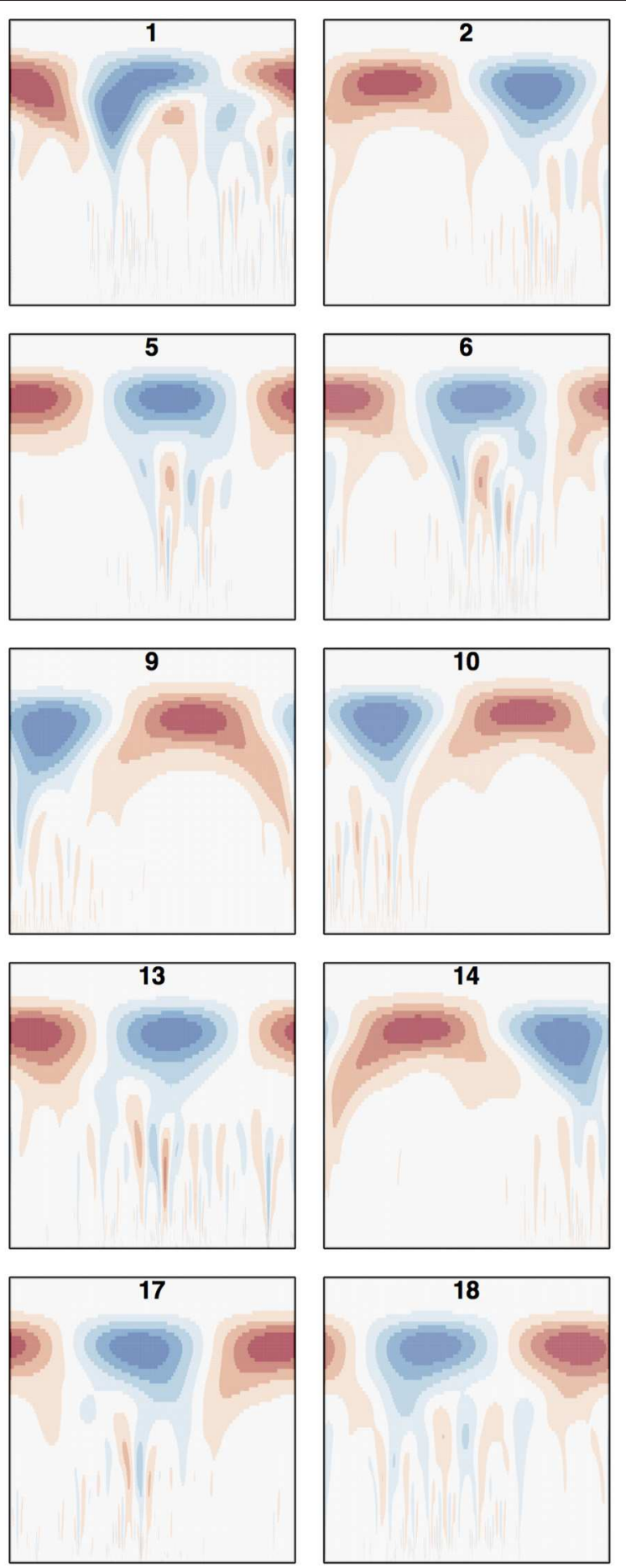
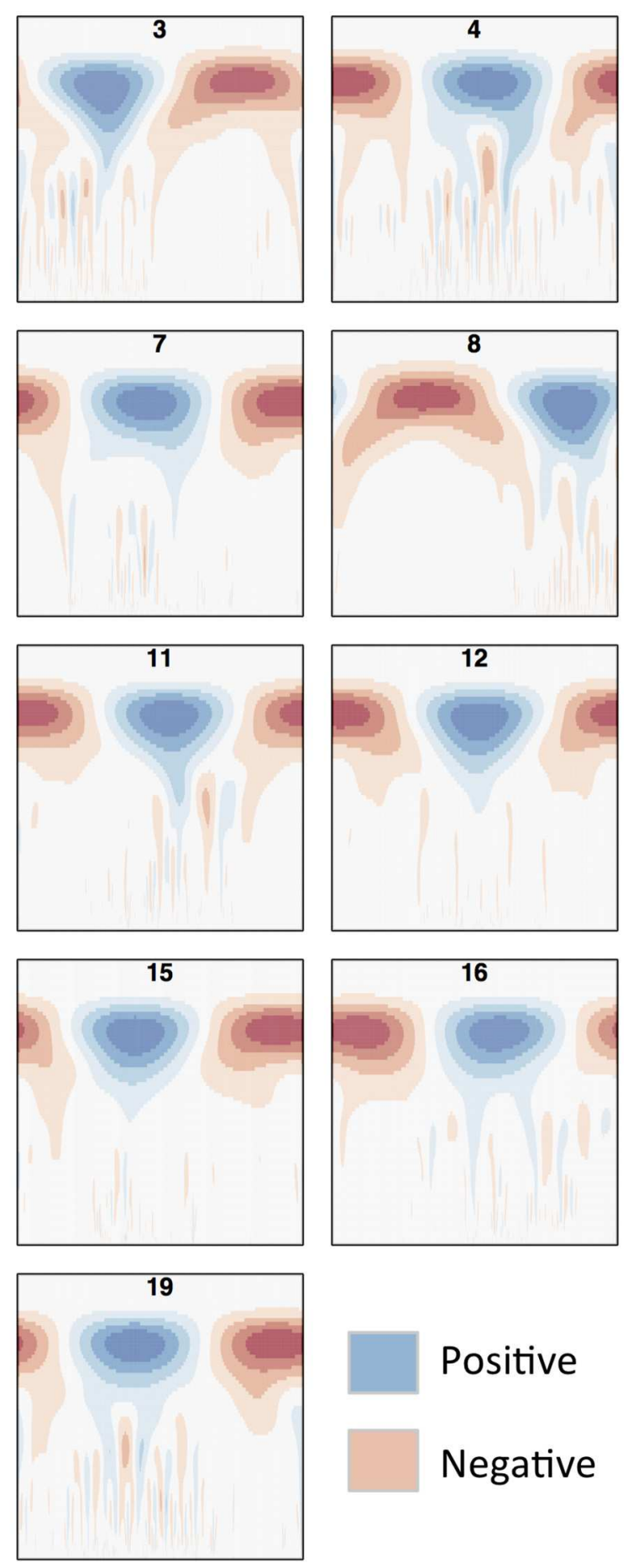

FIGURE 6 | Methylation (internode explant) CWT. Methylation (internode explant tissue) CWT landscapes of each $P$. trichocarpa chromosome. For each heatmap, the $\mathrm{x}$-axis represents position along the chromosome density signal $(\tau)$, the $y$-axis represents scale $(s)$ and each entry represents the wavelet coefficient $W(s, \tau)$. Positive coefficients are colored blue and indicate peaks, negative coefficients are colored red and indicate valleys. The "tooth-x-ray" centromeric signature is evident in many chromosomes, consisting of a broad-scale peak encompassing the centromeric/pericentromeric regions, and the lower scale valley within the large peak indicating the centromeric region. 


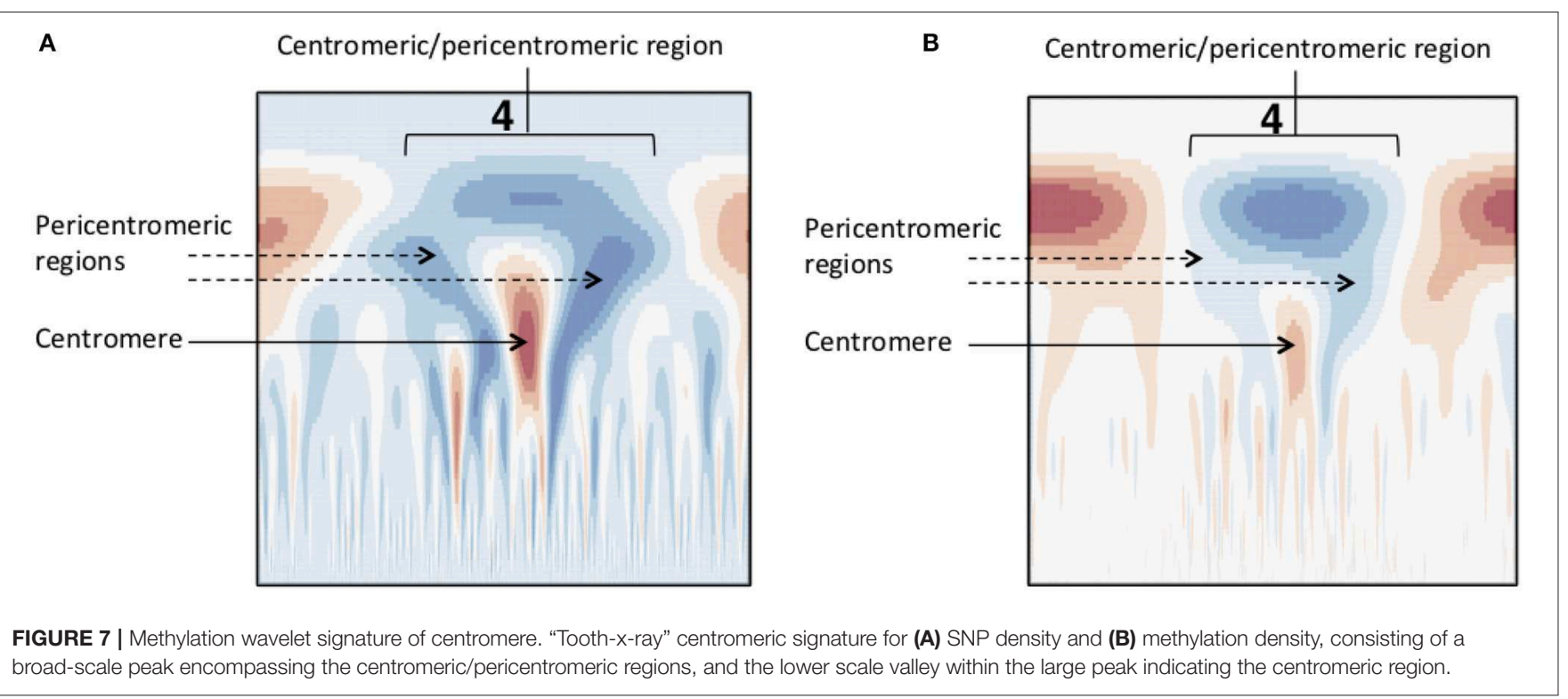

SNP density. However, this is not true for all chromosomes. The locations of highest genome gap density do not always coincide with the largest valley in SNP density, for example, in chromosome 12 (Figure S21), and the largest genome gaps do not always correspond to approximate centromere locations. Thus, the tooth-X-ray shape cannot be purely driven by genome gaps, and, as such, does not appear to be an artifact.

\subsection{Prediction of Centromere Position From Wavelet Coefficients}

Based on the knowledge of centromere signatures in the literature, and the CWT landscapes of gene, SNP and methylation profiles, we attempted to locate the position of the centromere on each $P$. trichocarpa chromosome by computationally identifying the characteristic tooth-X-ray shape in the CWT landscapes. Briefly, for each chromosome, we calculate the CWT of the scaled SNP density and methylation profiles, resulting in two coefficient landscapes. We identify the pericentromeric scale as the scale at which we find the maximum wavelet coefficient in the upper third of the methylation landscape, and identify the borders of the pericentromeric region as the zeroes of the wavelet vector on either side of the maximum coefficient. We then identify the centromeric scale as the minimum wavelet coefficient in the SNP wavelet landscape within the borders of the pericentromeric region, and then consider the approximate center of the centromere location to be the point of maximum difference between the methylation wavelet coefficients at the pericentromere scale and SNP wavelet coefficients at the centromere scale (Figure 8, Figure S1; Supplementary Note S1), and the general centromeric region borders as the points of intersection between the these two vectors on either side of the center (Table S1, yellow bars in Figure 8).

Mapping centromere repeats from various plants from the PGSB Repeat Element Catalog (Nussbaumer et al., 2012) as well as repeat sequences which were found to identify centromeres on certain $P$. trichocarpa chromosomes in a previous assembly (Cossu et al., 2012) using BLAST were consistent with the locations of centromeres identified using wavelet coefficients. Predicted centromere positions aligned well with the density profiles of repeat sequence BLAST hits, indicating that our centromere prediction strategy is likely identifying valid centromere positions (Figure 9). The waveletbased centromere identification through the use of multiple lines of evidence allows us to be more certain of centromeric regions, and also allows more specific locations to be identified than can be done by simply looking at repeat density, which map to broad regions of the genome. Layering multiple data types allows for the identification of putative centromere positions based on multiple lines of evidence, and thus, allows one to be more certain of their location.

Populus trichocarpa chromosomes contain homologous genome blocks, presumed to be derived from the salicoid genome duplication (Tuskan et al., 2006). Looking at the positions of predicted centromeres in Figures 8, 9, some paralogous chromosomes (see Tuskan et al., 2006) appear to have similar centromeric positions (for example, chromosomes 8 and 10, and chromosomes 12 and 15). This suggests that the current centromere positions potentially predate the salicoid duplication event. One can see in Figure 9 that certain PGSB peaks exist outside of predicted centromeric regions, suggesting centromere-like repeat sequences outside of the predicted active centromeric regions. These peaks outside of centromeric regions tend to overlap with syntenic blocks arising from a genome rearrangement involving a centromeric region (Figure 10, Figures S35, S36). For example, Figure 10A shows circos plots of all the syntenic blocks/homologous chromosome regions centered around chromosome 2. Centromeric regions predicted are shown as highlights on the chromosome ideogram. Visualizing only the syntenic blocks which overlap with centromeric regions (Figure 10B) provides information on the fate of active centromeres/centromeric 

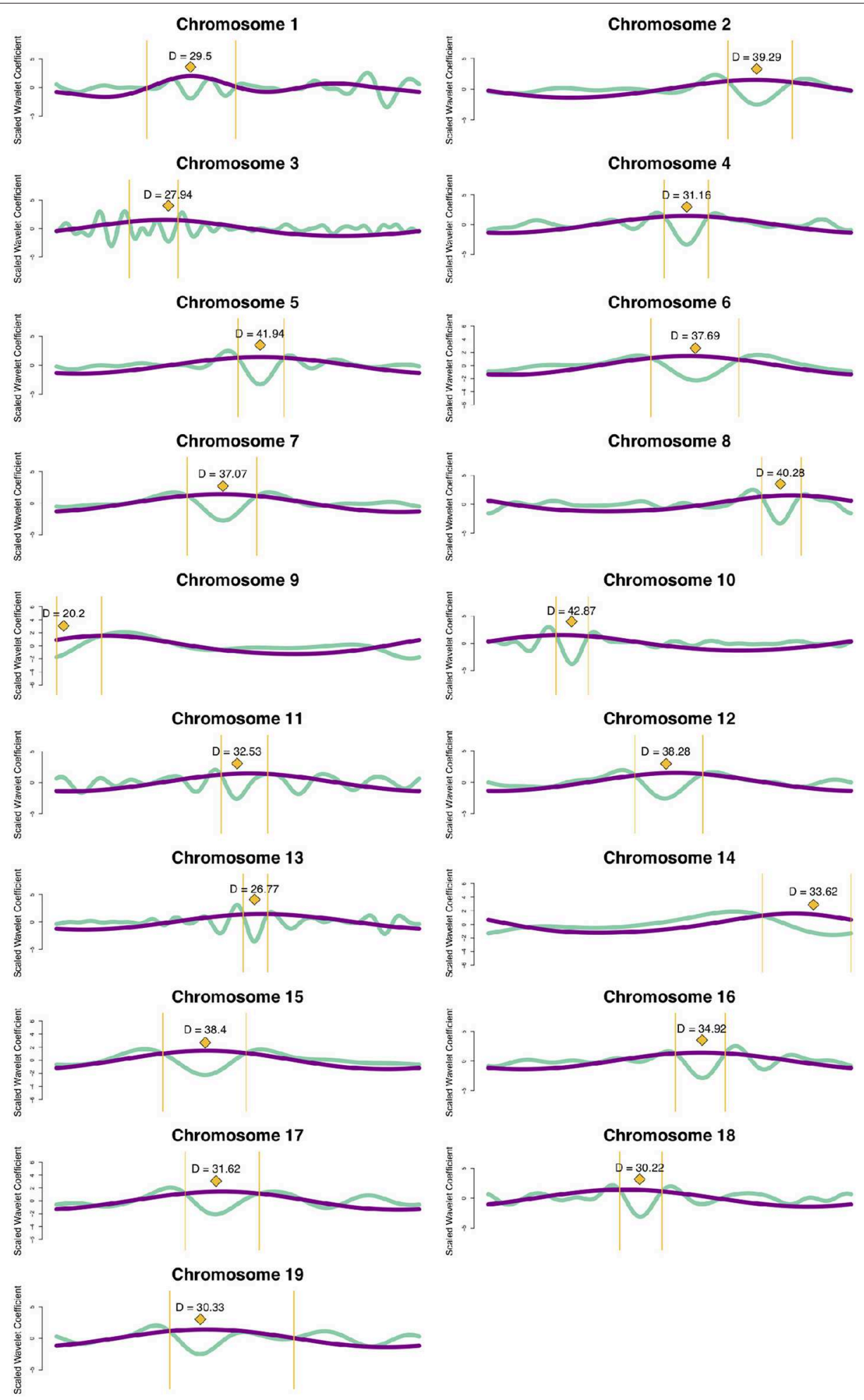

FIGURE 8 | Centromere positions. Line plots for each chromosome of methylation wavelet coefficients (internode explant tissue) at pericentromeric scale (purple lines) and SNP density wavelet coefficients at centromeric scale (green lines). Yellow diamonds represent the putative centromeric location, calculated as the point of maximum difference between the wavelet coefficients at these two scales. Yellow bars indicate the general centromeric region as the points of intersection between the two curves on either side of the centromeric region. The maximum difference $(D)$ between the unscaled wavelet coefficients used to determine the putative centromeric location (yellow diamonds) are shown for each chromosome. See Methods for further details. 


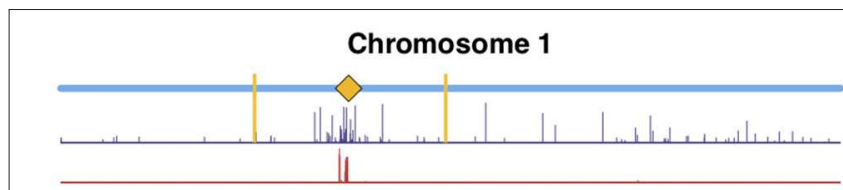

Chromosome 2

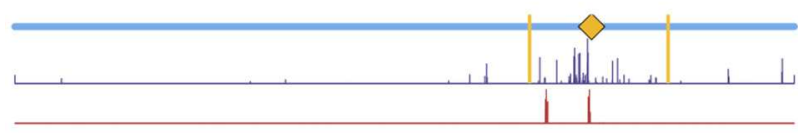

Chromosome 3

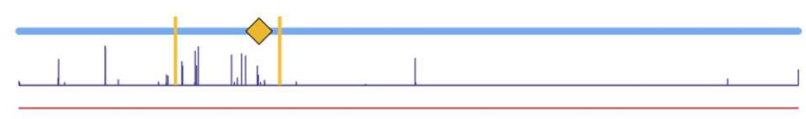

Chromosome 4

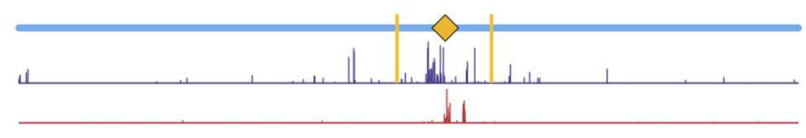

\section{Chromosome 5}

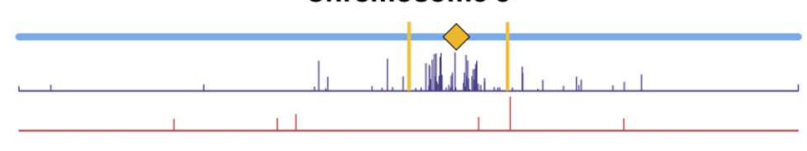

Chromosome 6

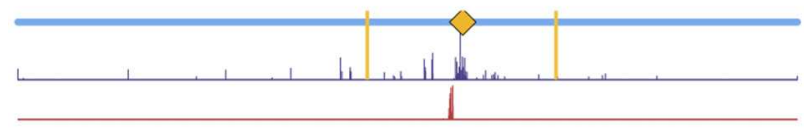

Chromosome 7

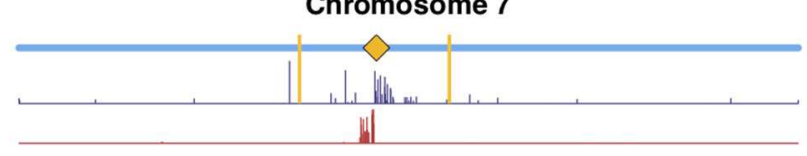

Chromosome 8

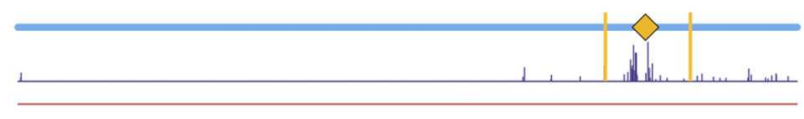

Chromosome 9

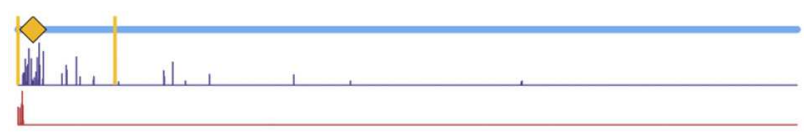

Chromosome 10

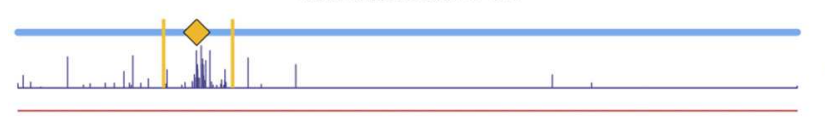

\section{Chromosome 11}

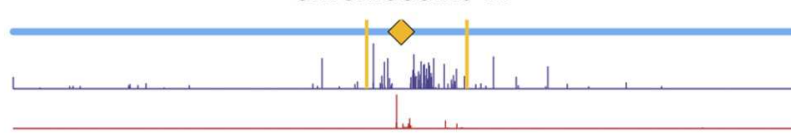

Chromosome 12

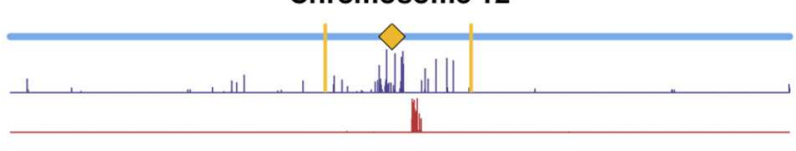

Chromosome 13

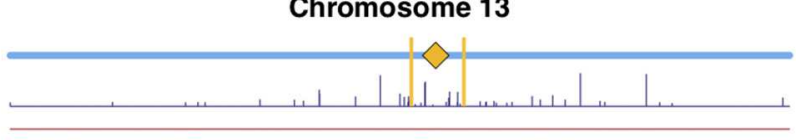

Chromosome 14

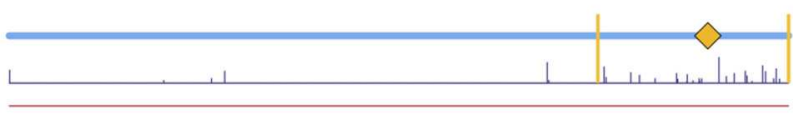

Chromosome 15

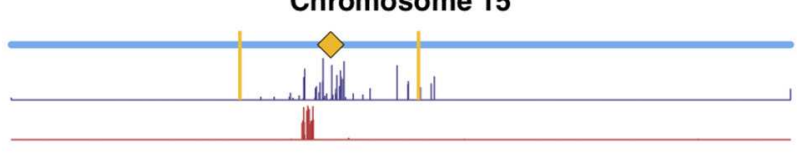

Chromosome 16

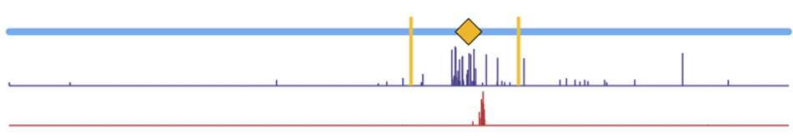

Chromosome 17

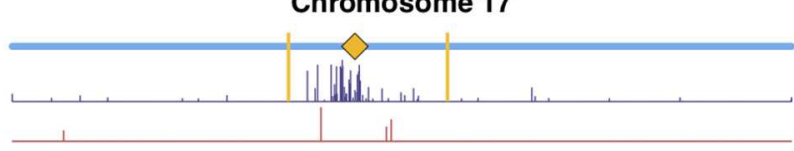

Chromosome 18

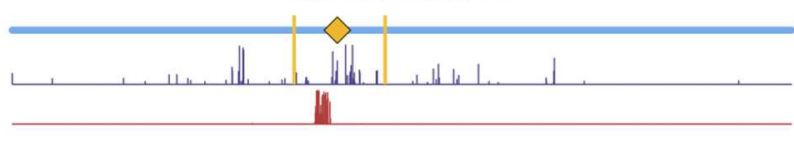

\section{Chromosome 19}

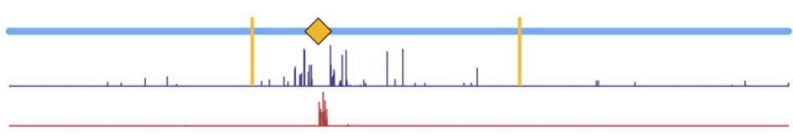

Lلl HL. PGSB centromere repeat BLAST match density I. Cossu et al. centromere repeat BLAST match density $\diamond$ Predicted centromere position

FIGURE 9 | Centromere positions and centromere repeats. Putative centromere positions (yellow diamonds) identified as in Figure 8 using methylation and SNP wavelet coefficients, as well as the density of BLAST matches of plant centromere repeat sequences (navy bars) and putative $P$. trichocarpa centromere repeat sequences (Cossu et al., 2012) (red bars). 

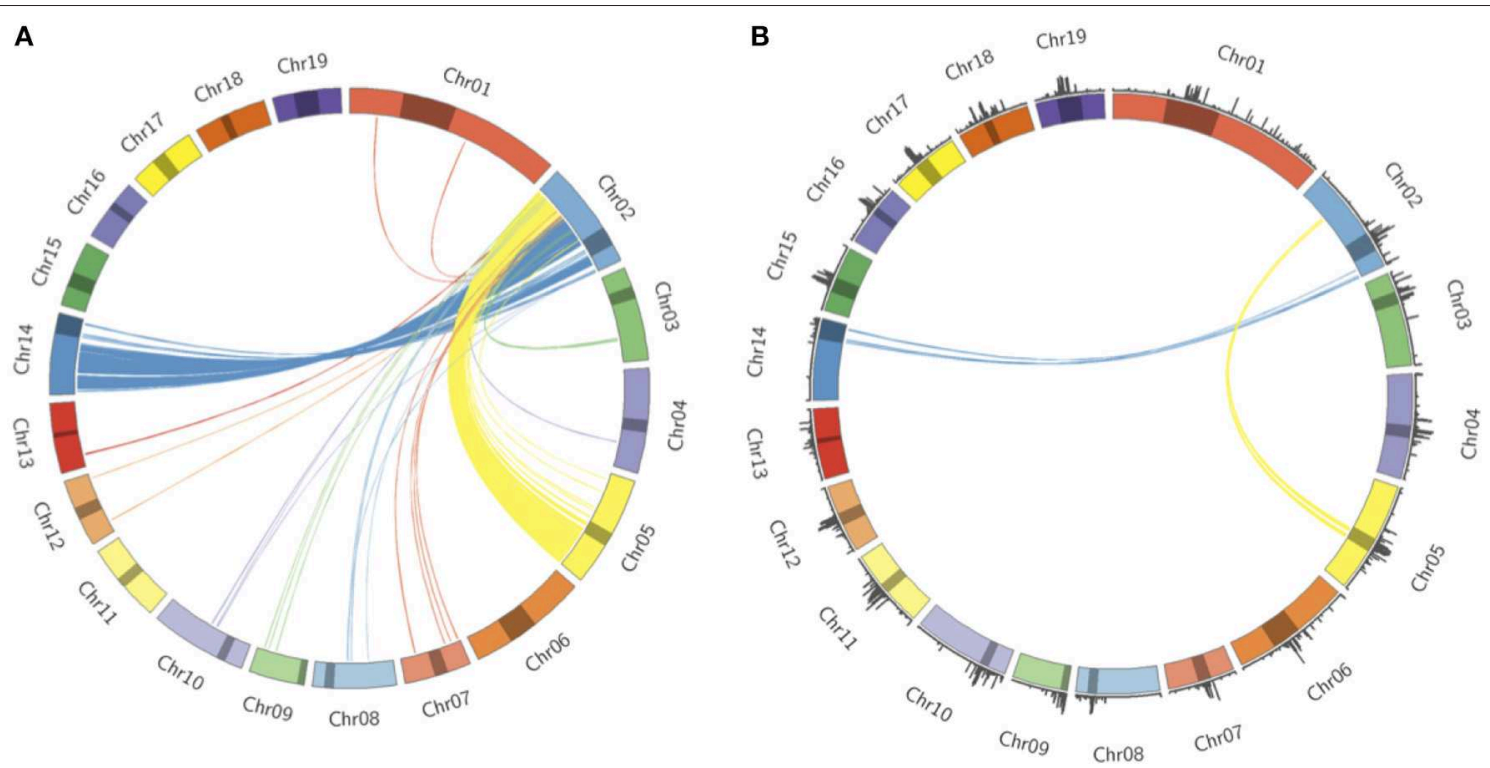

FIGURE 10 | Syntenic blocks and ancestral centromeric DNA. (A) Circos plot representing syntenic blocks involving homologous segments of DNA between chromosome two and other $P$. trichocarpa chromosomes. Chromosome lengths are represented on the ideogram with the predicted centromeric/pericentromeric regions shown as dark highlights. Links between chromosomes indicate homologous chromosome regions, and are colored according to the source chromosome, with chromosome 2 being the target chromosome. (B) Syntenic blocks which overlap with predicted centromeric/pericentromeric regions on source chromosomes. PGSB centromere repeat densities from Figure $\mathbf{9}$ are included as a bar chart on the ideogram.

DNA post-rearrangement. One can also see evidence for cases where the active centromere of a given chromosome segment was maintained after the chromosome rearrangement. The PGSB density plots from Figure 9 are shown as bar plots along the chromosome ideogram. Similar plots for other chromosomes can be seen in Figures S35, S36. These PGSB peaks representing centromere-like sequences outside of active centromere locations align well with syntenic blocks arising from centromeric locations, and can thus be interpreted as pieces of historic centromeric DNA from a genome duplication and subsequent genome rearrangement, known to occur in the history of $P$. trichocarpa.

\subsection{Co-evolution of Putative CENH3 With Centromeric Sequences}

The histone CENH3 epigenetically defines centromere position, and replaces normal histone $\mathrm{H} 3$ in the nucleosomes at the centromere (Watts et al., 2016). Silencing of this gene in Arabidopsis has been found to cause dwarfism, reduced mitotic divisions and sterility (Lermontova et al., 2011). CENH3 has been found to be adaptively evolving in Arabidopsis (Talbert et al., 2002). Analysis of CENH3 in various Brassicaceae showed that it is evolving adaptively at various sites which are potentially in contact with the centromeric DNA (Cooper and Henikoff, 2004). There is thus the hypothesis that CENH3 is co-evolving with the sequence of the centromere (Henikoff et al., 2001; Cooper and Henikoff, 2004). In a study involving a A. thaliana CENH3-null mutant expressing a Zea mays CENH3, it was found that while the Zea mays CENH3 localized to the same locations as endogenous A. thaliana CENH3, the Z. mays CENH3 centromeres were weaker, and resulted in genome elimination in crosses with wild-type $A$. thaliana (Maheshwari et al., 2017). Thus, the sequence of CENH3 could potentially have an impact on the strength of the centromere.

If the hypothesis of co-evolution between the CENH3 and centromeric sequences is true, one would expect to see correlations between Single Nucleotide Polymorphisms (SNPs) in $P$. trichocarpa CENH3 and P. trichocarpa centromeric regions. CENH3 is mostly a single copy in diploids, such as Arabidopsis (Watts et al., 2016) but there are some species that contain more than one copy. Wheat has two distinct copies of $\mathrm{CENH} 3$, and they seem to be evolutionarily divergent. They have different expression patterns, and one of them shows positive selection (Yuan et al., 2015). We identified two putative CENH3 genes in P. trichocarpa (Potri.014G096400 on chromosome 14 and Potri.002G169000 on chromosome 2) as BLAST (Altschul et al., 1990) matches of $A$. thaliana CENH3 (AT1G01370, for BLAST results see Table S2). It is interesting to note that chromosomes 2 and 14 are salicoid duplication paralogs. Of these two genes, Potri.014G096400 was annotated as being similar to a CENH3 gene, whereas Potri.002G169000 had no functional annotations. RNA-seq and EST information on Phytozome (Goodstein et al., 2012) confirmed that both of these genes are expressed (Figure S37). Expression information of these genes in the P. trichocarpa gene atlas on PhytoMine (Goodstein et al., 2012; Kalderimis et al., 2014) showed that the expression of these two genes varies across tissues, however, they are not coexpressed with one another (Figure 11). Both Potri.014G096400 and Potri.002G169000 genome sequences had multiple hits with CENH3 genes when BLASTed on NCBI. 


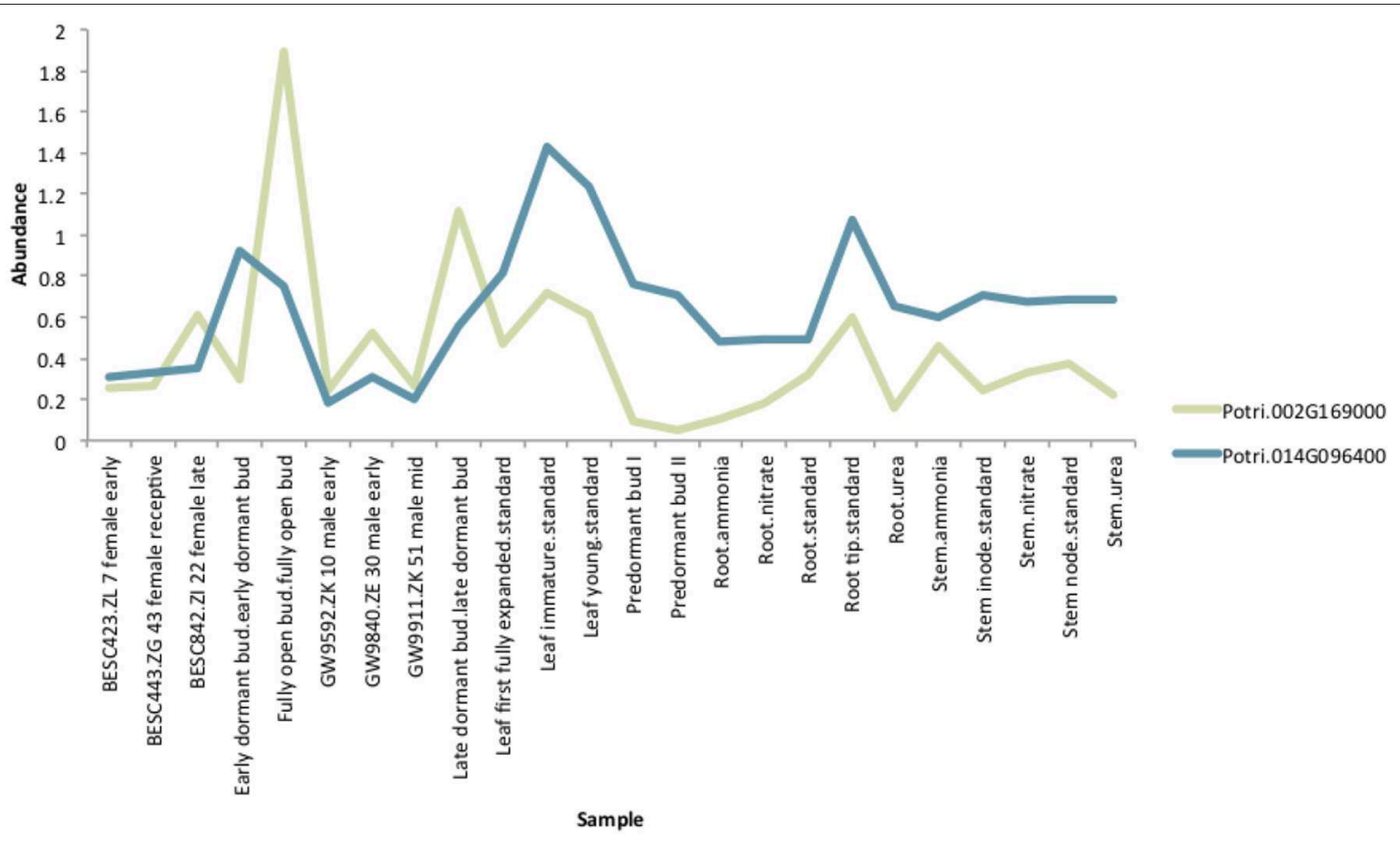

FIGURE 11 | CENH3 expression. Expression levels of putative P. trichocarpa CENH3 genes, Potri.002G169000 and Potri.014G096400. Expression data obtained from PhytoMine on Phytozome (Goodstein et al., 2012).

We determined correlations between all pairs of $\sim 10,000,000$ high confidence SNPs in a population of $882 P$. trichocarpa genotypes using the CCC metric (Climer et al., 2014a,b; Joubert et al., 2017) and extracted SNPs within Potri.014G096400 and Potri.002G169000 that had correlations with SNPs elsewhere in the genome (Tables S3, S4). When using a call rate constraint minimum of a 100 called alleles $(\sim 5 \%)$, a minimum overlap of 100 non-missing alleles in SNP correlations and requiring a minor allele frequency (MAF) $\geq 0.01$, we find concentrations of SNPs in the centromeric region of various chromosomes which are correlated with SNPs in Potri.002G169000 (Figure 12, Figure S38). We thus find strong evidence for the co-evolution for $\mathrm{CENH} 3$ with the centromeric sequences.

In particular, it appears that Potri.002G169000 seems to have a co-evolution signature with the centromere, much more so than Potri.014G096400, in that Potri.002G169000 contained SNPs correlating with 13 out of 19 centromeric regions, whereas Potri.014G096400 contained SNPs correlating with 5 out of 19 centromeric regions (centromeric regions in Table S1). While both Potri.002G169000 and Potri.014G096400 on average have more mutations than other $P$. trichocarpa histones (an expected phenomenon as CENH3 histones accumulate mutations faster than normal histones, as mentioned in Maheshwari et al., 2015), Potri.002G169000 contains more mutations than Potri.014G096400 (Figure S39; Table S5). Potri.014G096400 is also co-expressed with various other non-CENH3 histones, as well as a histone deacetylase and a histone methyltransferase on PhytoMine (Goodstein et al., 2012; Kalderimis et al., 2014) (Table S3), and the correlation neighborhood of Potri.002G169000 and Potri.014G096400 do not overlap at all (Figure 13; Tables S6, S7).
This seems to suggest that these two genes are functionally divergent. Given the facts that Potri.002G169000 has strong co-evolution signatures with the centromere (Figure 12) and Potri.014G096400 is co-expressing with non-CENH3 histones, one could hypothesize that Potri.002G169000 (a previously unannotated gene) is the primary functioning CENH3 in $P$. trichocarpa while Potri.014G096400 could be functioning more like a normal histone. If one looks at the position of SNPs within Potri.002G169000 and Potri.014G096400, it is evident that Potri.002G169000 contains more SNPs in transcribed regions of the gene that correlate with centromeric regions (Figure 14). In addition, Potri.002G169000 contains more SNPs in/near the histone domain that correlate with the centromere, when compared to Potri.014G096400. Potri.002G169000 also has more of the expected structure for a $\mathrm{CENH} 3$ gene, containing the histone domain in the $\mathrm{C}$ terminal, and having a variable $\mathrm{N}$ terminal domain (Watts et al., 2016).

Based on these various lines of evidence, we suggest that the previously unannotated Potri.002G169000 is the primary functioning CENH3 gene in P. trichocarpa.

\subsection{Concluding Remarks}

In this study we performed wavelet-based signal processing of multiple, heterogeneous data types to identify centromere positions and properties in $P$. trichocarpa. We found centromeres to be in gene-sparse regions, and found centromeric/pericentromeric regions to hypermethylated relative to the rest of the chromosomes, and found centromeric DNA to be hypomethylated relative to pericentromeric regions in many chromosomes across various tissues. The "tooth-X-ray" 


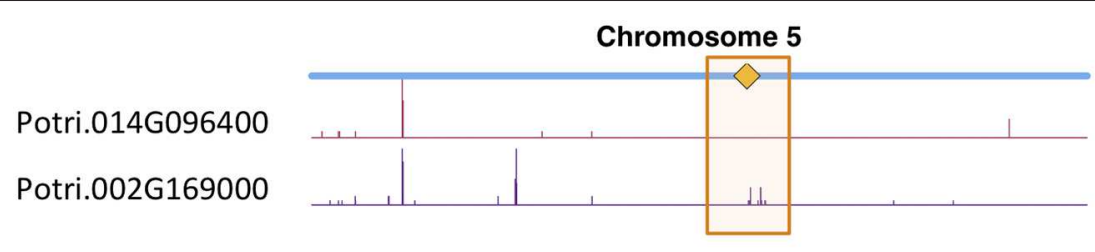

Chromosome 10

Potri.014G096400

Potri.002G169000

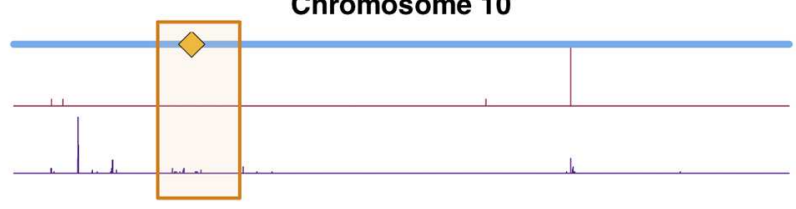

Chromosome 18

Potri.014G096400

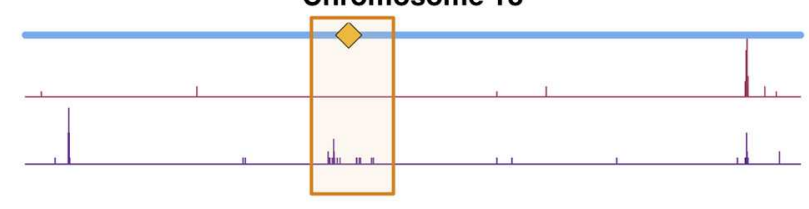

Potri.002G169000

Chromosome 19

Potri.014G096400

Potri.002G169000

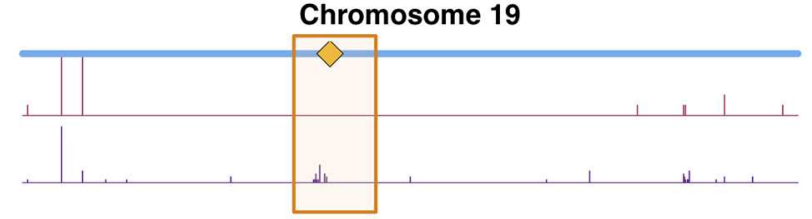

FIGURE 12 | CENH3 co-evolution. SNP density profiles across a selection of chromosomes involving SNPs which correlate with SNPs in putative CENH3 genes, Potri.002G169000 and Potri.014G096400 across a population of $P$. trichocarpa genotypes. One can clearly see the clusters of SNPs in the centromeric regions which are correlating with SNPs within these $\mathrm{CENH} 3$ genes.

wavelet signature was identified as a characteristic signature of the centromere in the wavelet landscapes of SNP density profiles. The use of wavelet coefficients allowed us to identify the approximate centromeric locations. These locations were supported by mapping of repeat sequences, and could be further validated through experimental techniques, such as ChIP (chromatin immunoprecipitation)-Seq. We also found evidence for the co-evolution of the sequence of the centromere-specific histone CENH3 with the sequences of the centromere on many chromosomes. In particular, we found that the previously unannotated gene Potri.002G169000 is the most likely candidate for an active, centromere-co-evolving $\mathrm{CENH} 3$ gene in $P$. trichocarpa and not the currently annotated CENH3 gene, Potri.014G096400.

This study illustrates how through integrating multiple sources of data, one can arrive at a more comprehensive understanding of the system one is investigating. In this case, we have produced at a more reliable and detailed characterization of centromere location in P. trichocarpa, involving information from multiple 'omics data layers and providing information about centromeric signatures derived from multiple sources. In order to extend these analyses to produce an automated centromere prediction approach applicable to other species, further testing and validation will be required. The wavelet-based approach

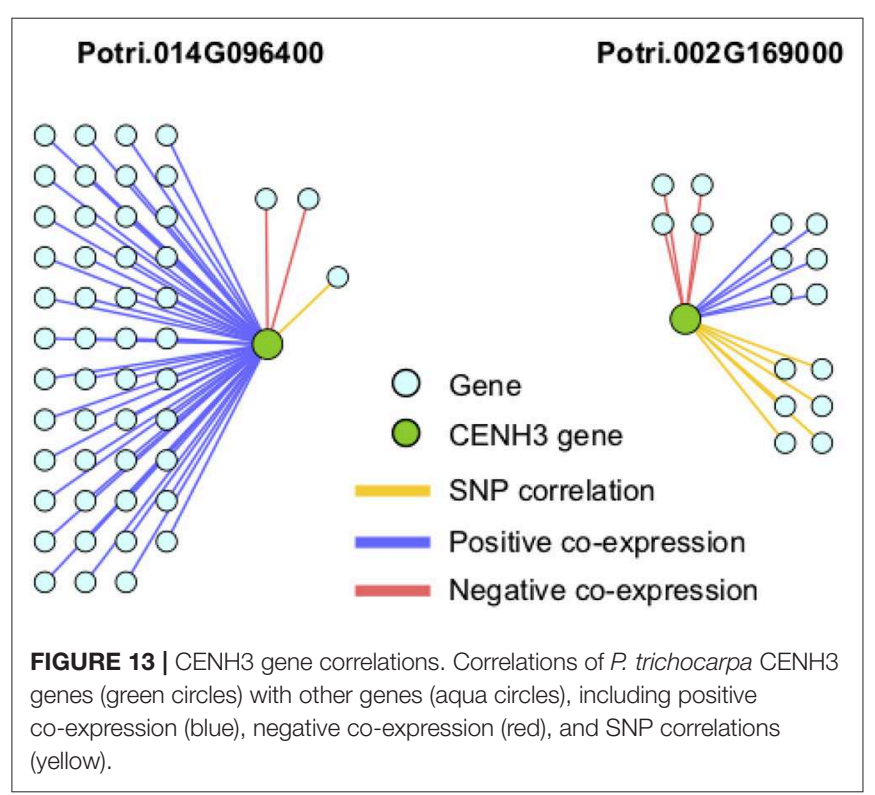

would need to be applied to multiple species, and validated against exact centromeric locations determined using ChIP-seq. 


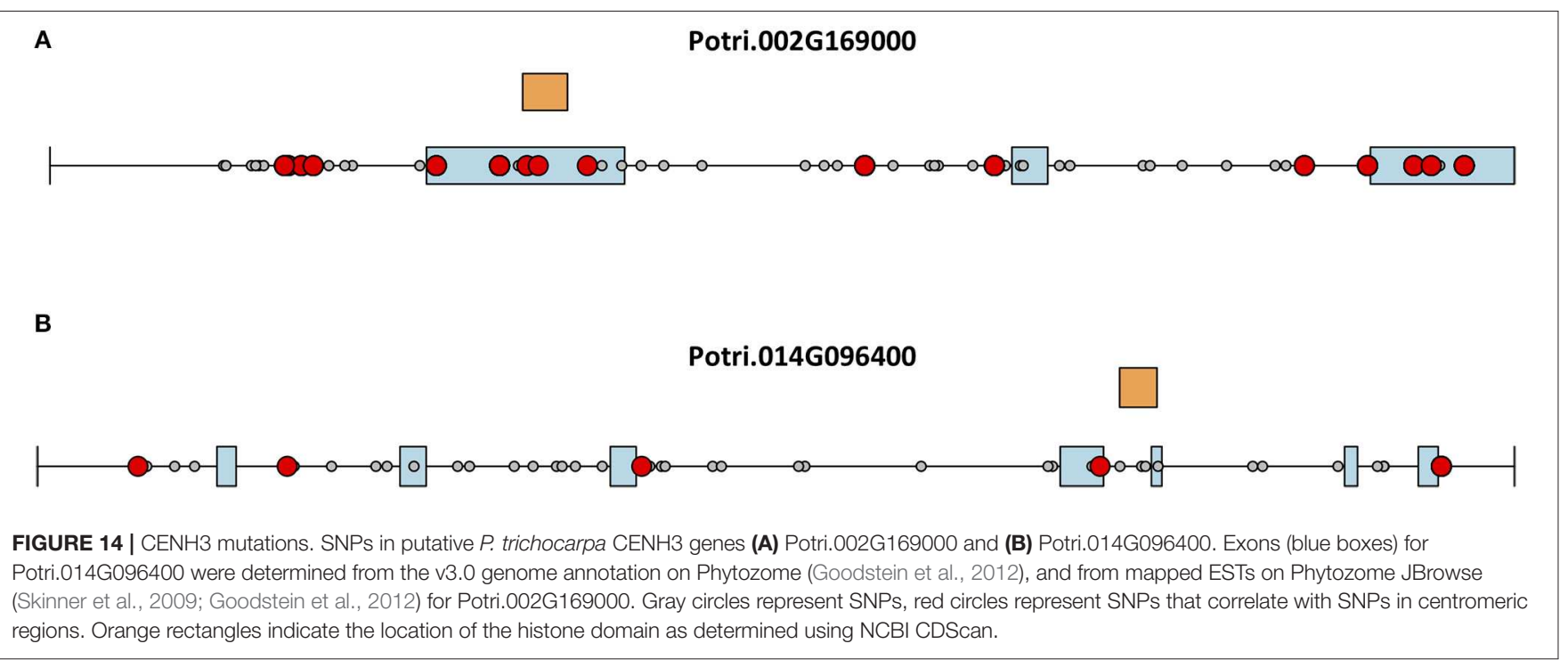

This study illustrated the utility of wavelet-based signal processing of genomic signals to identify structural characteristics of chromosomes. While this study made primary use of the larger-scale wavelet coefficients, we would recommend the use of the smaller scale wavelet coefficients to investigate smaller-scale structural characteristics, such as nucleosome occupancy.

\section{AUTHOR CONTRIBUTIONS}

DW and DJ conceived of and designed the study. DW performed the analysis, developed the wavelet centromere identification strategy, interpreted the results, and wrote the manuscript. GT lead the sequencing of Populus genotypes. SD and DM-S performed the SNP calling. JS and AS contributed the genome sequence and transcriptome expression analysis. MS mapped gene expression atlas reads and calculated gene expression TPM values. WJ developed the parallel GPU CCC metric code. DW, DJ, GT, DM-S, and AS edited the manuscript.

\section{FUNDING}

Funding provided by The BioEnergy Science Center (BESC) and The Center for Bioenergy Innovation (CBI). U.S. Department of Energy Bioenergy Research Centers supported by the Office of Biological and Environmental Research in the DOE Office of Science.

This research was also supported by the Plant-Microbe Interfaces Scientific Focus Area (http://pmi.ornl.gov) in the Genomic Science Program, the Office of Biological and Environmental Research (BER) in the U.S. Department of Energy Office of Science, and by the Department of Energy, Laboratory Directed Research and Development funding (7758), at the Oak Ridge National Laboratory. Oak Ridge National Laboratory is managed by UT-Battelle, LLC, for the US DOE under contract DE-AC05-00OR22725.
An award of computer time was provided by the INCITE program. This research also used resources of the Oak Ridge Leadership Computing Facility, which is a DOE Office of Science User Facility supported under Contract DE-AC05-00OR22725. This research also used resources of the Compute and Data Environment for Science (CADES) at the Oak Ridge National Laboratory, which is supported by the Office of Science of the U.S. Department of Energy under Contract No. DEAC05-00OR22725.

Support for the Poplar GWAS dataset was provided by The BioEnergy Science Center (BESC) and The Center for Bioenergy Innovation (CBI). U.S. Department of Energy Bioenergy Research Centers supported by the Office of Biological and Environmental Research in the DOE Office of Science The Poplar GWAS Project used resources of the Oak Ridge Leadership Computing Facility and the Compute and Data Environment for Science at Oak Ridge National Laboratory, which is supported by the Office of Science of the U.S. Department of Energy under Contract No. DE-AC05-00OR22725.

The work conducted by the U.S. Department of Energy Joint Genome Institute is supported by the Office of Science of the U.S. Department of Energy under Contract No. DE-AC02$05 \mathrm{CH} 11231$.

\section{ACKNOWLEDGMENTS}

The authors would like to acknowledge the Department of Energy Joint Genome Institute (JGI) for the sequencing of $P$. trichocarpa genomes, as well as Ryan McCormick, Sandra Truong, and Anna Furches for useful discussions about the manuscript.

\section{SUPPLEMENTARY MATERIAL}

The Supplementary Material for this article can be found online at: https://www.frontiersin.org/articles/10.3389/fgene. 2019.00487/full\#supplementary-material 


\section{REFERENCES}

Altschul, S. F., Gish, W., Miller, W., Myers, E. W., and Lipman, D. J. (1990). Basic local alignment search tool. J. Mol. Biol. 215, 403-410.

Barnett, D. W., Garrison, E. K., Quinlan, A. R., Strömberg, M. P., and Marth, G. T. (2011). BamTools: a C++ API and toolkit for analyzing and managing BAM files. Bioinformatics 27, 1691-1692. doi: 10.1093/bioinformatics/btr174

Bekele, W. A., Wieckhorst, S., Friedt, W., and Snowdon, R. J. (2013). Highthroughput genomics in sorghum: from whole-genome resequencing to a snp screening array. Plant Biotechnol. J. 11, 1112-1125. doi: 10.1111/pbi.12106

Climer, S., Templeton, A. R., and Zhang, W. (2014a). Allele-specific network reveals combinatorial interaction that transcends small effects in Psoriasis GWAS. PLoS Comput. Biol. 10:e1003766. doi: 10.1371/journal.pcbi.1003766

Climer, S., Yang, W., Fuentes, L., Dávila-Román, V. G., and Gu, C. C. (2014b). A custom correlation coefficient (CCC) approach for fast identification of multiSNP association patterns in genome-wide SNPs data. Genet. Epidemiol. 38, 610-621. doi: 10.1002/gepi.21833

Constantine, W., Hesterberg, T., Wittkowski, K., Song, T., and Kaluzny, S. (2016). splus2R: Supplemental S-PLUS Functionality in R. R package version 1.2-2.

Constantine, W., and Percival, D. (2016). wmtsa: Wavelet Methods for Time Series Analysis. R package version 2.0-2.

Cooper, J. L., and Henikoff, S. (2004). Adaptive evolution of the histone fold domain in centromeric histones. Mol. Biol. Evol. 21, 1712-1718. doi: $10.1093 / \mathrm{molbev} / \mathrm{msh} 179$

Copenhaver, G. P., Nickel, K., Kuromori, T., Benito, M.-I., Kaul, S., Lin, X., et al. (1999). Genetic definition and sequence analysis of arabidopsis centromeres. Science 286, 2468-2474.

Cossu, R. M., Buti, M., Giordani, T., Natali, L., and Cavallini, A. (2012). A computational study of the dynamics of LTR retrotransposons in the populus trichocarpa genome. Tree Genet. Genomes 8, 61-75. doi: 10.1007/s11295-011-0421-3

Danecek, P., Auton, A., Abecasis, G., Albers, C. A., Banks, E., DePristo, M. A., et al. (2011). The variant call format and VCFtools. Bioinformatics 27, 2156-2158. doi: 10.1093/bioinformatics/btr330

Evans, L. M., Slavov, G. T., Rodgers-Melnick, E., Martin, J., Ranjan, P., Muchero, W., et al. (2014). Population genomics of Populus trichocarpa identifies signatures of selection and adaptive trait associations. Nat. Genet. 46, 10891096. doi: 10.1038/ng.3075

Feng, C., Liu, Y., Su, H., Wang, H., Birchler, J., and Han, F. (2015). Recent advances in plant centromere biology. Sci. China Life Sci. 58, 240-245. doi: 10.1007/s11427-015-4818-3

Furuyama, S., and Biggins, S. (2007). Centromere identity is specified by a single centromeric nucleosome in budding yeast. Proc. Natl. Acad. Sci. U.S.A. 104, 14706-14711. doi: 10.1073/pnas.0706985104

Gomez-Cabrero, D., Abugessaisa, I., Maier, D., Teschendorff, A., Merkenschlager, M., Gisel, A. J., et al, (2014). Data integration in the era of omics: current and future challenges. BMC Syst. Biol. 8:I1. doi: 10.1186/1752-0509-8-S2-I1

Goodstein, D. M., Shu, S., Howson, R., Neupane, R., Hayes, R. D., Fazo, J., et al. (2012). Phytozome: a comparative platform for green plant genomics. Nucleic Acids Res. 40, D1178-D1186. doi: 10.1093/nar/gkr944

Grigoriev, I. V., Nordberg, H., Shabalov, I., Aerts, A., Cantor, M., Goodstein, D., et al. (2011). The genome portal of the Department of Energy Joint Genome Institute. Nucleic Acids Res. 40, 1-7. doi: 10.1093/nar/gkr947

Haug-Baltzell, A., Stephens, S. A., Davey, S., Scheidegger, C. E., and Lyons, E. (2017). SynMap2 and SynMap3D: web-based whole-genome synteny browsers. Bioinformatics 33, 2197-2198. doi: 10.1093/bioinformatics/btx144

Henikoff, S., Ahmad, K., and Malik, H. S. (2001). The centromere paradox: stable inheritance with rapidly evolving dNA. Science 293, 1098-1102. doi: $10.1126 /$ science. 1062939

Joubert, W., Nance, J., Climer, S., Weighill, D., and Jacobson, D. (2017). Parallel accelerated custom correlation coefficient calculations for genomics applications. Parallel Comput. 84, 15-23. doi: 10.1016/j.parco.2019.02.003

Kalderimis, A., Lyne, R., Butano, D., Contrino, S., Lyne, M., Heimbach, J., et al. (2014). Intermine: extensive web services for modern biology. Nucleic Acids Res. 42, W468-W472. doi: 10.1093/nar/gku301

Krzywinski, M. I., Schein, J. E., Birol, I., Connors, J., Gascoyne, R., Horsman, D., et al. (2009). Circos: an information aesthetic for comparative genomics. Genome Res. 19, 1639-1645. doi: 10.1101/gr.092759.109
Leavey, C., James, M., Summerscales, J., and Sutton, R. (2003). An introduction to wavelet transforms: a tutorial approach. Insight Non Destruct. Testing Condit. Monit. 45, 344-353. doi: 10.1784/insi.45.5.344.52875

Lermontova, I., Koroleva, O., Rutten, T., Fuchs, J., Schubert, V., Moraes, I., et al. (2011). Knockdown of CENH3 in arabidopsis reduces mitotic divisions and causes sterility by disturbed meiotic chromosome segregation. Plant J. 68, 40-50. doi: 10.1111/j.1365-313X.2011.04664.x

Li, H., Handsaker, B., Wysoker, A., Fennell, T., Ruan, J., Homer, N., et al. (2009). The sequence alignment/map format and SAMtools. Bioinformatics 25, 20782079. doi: 10.1093/bioinformatics/btp352

Liang, D., Zhang, Z., Wu, H., Huang, C., Shuai, P., Ye, C.-Y., et al. (2014). Single-base-resolution methylomes of populus trichocarpa reveal the association between dna methylation and drought stress. BMC Genet. 15:S9. doi: 10.1186/1471-2156-15-S1-S9

Lyons, E., Pedersen, B., Kane, J., and Freeling, M. (2008). The value of nonmodel genomes and an example using SynMap within CoGe to dissect the hexaploidy that predates the rosids. Trop. Plant Biol. 1, 181-190. doi: 10.1007/s12042-008-9017-y

Machado, J. T., Costa, A. C., and Quelhas, M. D. (2011). Wavelet analysis of human DNA. Genomics 98, 155-163. doi: 10.1016/j.ygeno.2011.05.010

Maheshwari, S., Ishii, T., Brown, C. T., Houben, A., and Comai, L. (2017). Centromere location in arabidopsis is unaltered by extreme divergence in CENH3 protein sequence. Genome Res. 27, 471-478. doi: 10.1101/gr.214619.116

Maheshwari, S., Tan, E. H., West, A., Franklin, F. C. H., Comai, L., and Chan, S. W. (2015). Naturally occurring differences in CENH3 affect chromosome segregation in zygotic mitosis of hybrids. PLoS Genet. 11:e1004970. doi: 10.1371/journal.pgen.1004970

Marchler-Bauer, A., and Bryant, S. H. (2004). CD-search: protein domain annotations on the fly. Nucleic Acids Res. 32(Suppl._2), W327-W331. doi: $10.1093 /$ nar/gkh454

Marchler-Bauer, A., Derbyshire, M. K., Gonzales, N. R., Lu, S., Chitsaz, F., Geer, L. Y., et al. (2014). CDD: Ncbi's conserved domain database. Nucleic Acids Res. 43, D222-D226. doi: 10.1093/nar/gku1221

Marchler-Bauer, A., Lu, S., Anderson, J. B., Chitsaz, F., Derbyshire, M. K., DeWeese-Scott, C., et al. (2010). CDD: a conserved domain database for the functional annotation of proteins. Nucleic Acids Res. 39(Suppl._1), D225-D229. doi: $10.1093 /$ nar/gkq1189

McCormick, R. F., Truong, S. K., Sreedasyam, A., Jenkins, J., Shu, S., Sims, D., et al. (2017). The Sorghum bicolor reference genome: improved assembly and annotations, a transcriptome atlas, and signatures of genome organization. bioRxiv 110593. doi: 10.1101/110593

Mehrotra, S., and Goyal, V. (2014). Repetitive sequences in plant nuclear DNA: types, distribution, evolution and function. Genomics Proteomics Bioinformatics 12, 164-171. doi: 10.1016/j.gpb.2014.07.003

Neph, S., Kuehn, M. S., Reynolds, A. P., Haugen, E., Thurman, R. E., Johnson, A. K., et al. (2012). BEDOPS: high-performance genomic feature operations. Bioinformatics 28, 1919-1920. doi: 10.1093/bioinformatics/b ts277

Neuwirth, E. (2014). RColorBrewer: ColorBrewer Palettes. R package version 1.1-2.

Nordberg, H., Cantor, M., Dusheyko, S., Hua, S., Poliakov, A., Shabalov, I., et al. (2014). The genome portal of the Department of Energy Joint Genome Institute: 2014 updates. Nucleic Acids Res. 42, D26-D31. doi: 10.1093/nar/gkt1069

Nussbaumer, T., Martis, M. M., Roessner, S. K., Pfeifer, M., Bader, K. C., Sharma, S., et al. (2012). MIPS plantsDB: a database framework for comparative plant genome research. Nucleic Acids Res. 41, D1144-D1151. doi: $10.1093 / \mathrm{nar} / \mathrm{gks} 1153$

Nychka, D., Furrer, R., Paige, J., and Sain, S. (2017). Fields: Tools for Spatial Data. $\mathrm{R}$ package version 9.6 .

O'Connor, C. (2008). Chromosome segregation in mitosis: the role of centromeres. Nat. Educ. 1:28.

Ossowski, S., Schneeberger, K., Lucas-Lledó, J. I., Warthmann, N., Clark, R. M., Shaw, R. G., et al. (2010). The rate and molecular spectrum of spontaneous mutations in arabidopsis thaliana. Science 327, 92-94. doi: $10.1126 /$ science. 1180677

Percival, D. B., and Walden, A. T. (2006). Wavelet Methods for Time Series Analysis, Vol. 4. New York, NY: Cambridge University Press. 
Pinosio, S., Giacomello, S., Faivre-Rampant, P., Taylor, G., Jorge, V., Le Paslier, M. C., et al. (2016). Characterization of the poplar pan-genome by genomewide identification of structural variation. Mol. Biol. Evol. 33, 2706-2719. doi: $10.1093 / \mathrm{molbev} / \mathrm{msw} 161$

Purcell, S., Neale, B., Todd-Brown, K., Thomas, L., Ferreira, M. A., Bender, D., et al. (2007). Plink: a tool set for whole-genome association and population-based linkage analyses. Am. J. Hum. Genet. 81, 559-575. doi: 10.1086/519795

Quinlan, A. R. (2014). Bedtools: the swiss-army tool for genome feature analysis. Curr. Protoc. Bioinformatics 47, 11-12. doi: 10.1002/0471250953.bi1112s47

R Core Team (2015). R: A Language and Environment for Statistical Computing. Vienna: R Foundation for Statistical Computing.

RStudio Team (2016). RStudio: Integrated Development Environment for R. Boston, MA: RStudio, Inc.

Shannon, P., Markiel, A., Ozier, O., Baliga, N. S., Wang, J. T., Ramage, D., et al. (2003). Cytoscape: a software environment for integrated models of biomolecular interaction networks. Genome Res. 13, 2498-2504. doi: $10.1101 /$ gr. 1239303

Skinner, M. E., Uzilov, A. V., Stein, L. D., Mungall, C. J., and Holmes, I. H. (2009). JBrowse: A next-generation genome browser. Genome Res. 19, 1630-1638. doi: 10.1101/gr.094607.109

Slavov, G. T., DiFazio, S. P., Martin, J., Schackwitz, W., Muchero, W., RodgersMelnick, E., et al. (2012). Genome resequencing reveals multiscale geographic structure and extensive linkage disequilibrium in the forest tree Populus trichocarpa. New Phytol. 196, 713-725. doi: 10.1111/j.1469-8137.2012.04258.x

Spencer, C. C., Deloukas, P., Hunt, S., Mullikin, J., Myers, S., Silverman, B., et al. (2006). The influence of recombination on human genetic diversity. PLoS Genet. 2:e148. doi: 10.1371/journal.pgen.0020148

Talbert, P. B., Masuelli, R., Tyagi, A. P., Comai, L., and Henikoff, S. (2002). Centromeric localization and adaptive evolution of an arabidopsis histone $\mathrm{H} 3$ variant. Plant Cell 14, 1053-1066. doi: 10.1105/tpc.010425

Tange, O. (2011). GNU parallel-the command-line power tool. USENIX Mag. 36, $42-47$.

Tuskan, G., Slavov, G., DiFazio, S., Muchero, W., Pryia, R., Schackwitz, W., et al. (2011). Populus resequencing: towards genome-wide association studies. BMC Proc. 5:I21. doi: 10.1186/1753-6561-5-S7-I21

Tuskan, G. A., Difazio, S., Jansson, S., Bohlmann, J., Grigoriev, I., Hellsten, U., et al. (2006). The Genome of Black Cottonwood, Populus trichocarpa (Torr. \& Gray). Science 313, 1596-1604. doi: 10.1126/science.11 28691

Vining, K. J., Pomraning, K. R., Wilhelm, L. J., Priest, H. D., Pellegrini, M., Mockler, T. C., et al. (2012). Dynamic DNA cytosine methylation in the Populus trichocarpa genome: tissue-level variation and relationship to gene expression. BMC Genomics 13:27. doi: 10.1186/1471-2164-13-27
Watts, A., Kumar, V., and Bhat, S. R. (2016). Centromeric histone H3 protein: from basic study to plant breeding applications. J. Plant Biochem. Biotechnol. 25, 339-348. doi: 10.1007/s13562-016-0368-4

Weighill, D. A., Jones, P., Shah, M., Ranjan, P., Muchero, W., Schmutz, J., et al. (2018). Pleiotropic and epistatic network-based discovery: Integrated networks for target gene discovery. Front. Energy Res. 6:30. doi: 10.3389/fenrg.2018.00030

$\mathrm{Wu}, \mathrm{Q}$, and Castleman, K. R. (2000). "Automated chromosome classification using wavelet-based band pattern descriptors," in Computer-Based Medical Systems, 2000. CBMS 2000. Proceedings. 13th IEEE Symposium on (Houston, TX: IEEE), 189-194.

Yuan, J., Guo, X., Hu, J., Lv, Z., and Han, F. (2015). Characterization of two CENH3 genes and their roles in wheat evolution. New Phytol. 206, 839-851. doi: $10.1111 /$ nph. 13235

Zhang, W., Lee, H.-R., Koo, D.-H., and Jiang, J. (2008). Epigenetic modification of centromeric chromatin: hypomethylation of dna sequences in the cenh3associated chromatin in arabidopsis thaliana and maize. Plant Cell 20, 25-34. doi: $10.1105 /$ tpc. 107.057083

Zhang, X., Yazaki, J., Sundaresan, A., Cokus, S., Chan, S. W.-L., Chen, H., et al. (2006). Genome-wide high-resolution mapping and functional analysis of dna methylation in arabidopsis. Cell 126, 1189-1201. doi: 10.1016/j.cell.2006.08.003

Disclaimer: This manuscript has been authored by UT-Battelle, LLC under Contract No. DE-AC05-00OR22725 with the U.S. Department of Energy. The United States Government retains and the publisher, by accepting the article for publication, acknowledges that the United States Government retains a nonexclusive, paid-up, irrevocable, worldwide license to publish or reproduce the published form of this manuscript, or allow others to do so, for United States Government purposes. The Department of Energy will provide public access to these results of federally sponsored research in accordance with the DOE Public Access Plan (http://energy.gov/downloads/doe-public-access-plan).

Conflict of Interest Statement: The authors declare that the research was conducted in the absence of any commercial or financial relationships that could be construed as a potential conflict of interest.

Copyright (C) 2019 Weighill, Macaya-Sanz, DiFazio, Joubert, Shah, Schmutz, Sreedasyam, Tuskan and Jacobson. This is an open-access article distributed under the terms of the Creative Commons Attribution License (CC BY). The use, distribution or reproduction in other forums is permitted, provided the original author(s) and the copyright owner(s) are credited and that the original publication in this journal is cited, in accordance with accepted academic practice. No use, distribution or reproduction is permitted which does not comply with these terms. 